\title{
The complete determination of wide Richaud-Degert types which are not 5 modulo 8 with class number one
}

\author{
by \\ JungYun LeE (Daejeon)
}

1. Introduction and statement of results. Let $d$ be a square free integer and $h(d)$ be the class number of $\mathbb{Q}(\sqrt{d})$. Let $d=n^{2}+r$ be a square free integer such that $r \mid 4 n$ and $-n<r \leq n$. In this case, we call $d$ a Richaud-Degert type. If $|r| \neq 1,4$, then it is called a wide Richaud-Degert type, while if $|r|=1$ or 4 , then it is called a narrow Richaud-Degert type.

There had been many conjectures about the upper bound of RichaudDegert types $d$ with $h(d)=1$. Yokoi [15] conjectured that $h\left(n^{2}+4\right)>1$ if $n>17$. Chowla [7] conjectured that $h\left(4 n^{2}+1\right)>1$ if $n>13$. Biró [1], [2] proved the above two conjectures. Also Mollin [10] conjectured that $h\left(n^{2}-4\right)=1$ if $n>21$. Mollin's conjecture was confirmed by Byeon, Kim and the author [5]. This determines all real quadratic fields of narrow Richaud-Degert types with class number 1.

In this paper, we prove the following Mollin-Williams conjecture (see Conjecture 5.4.4 on page 176 of [11]). From this, we show that there are exactly 14 wide R-D types $d \not \equiv 5(\bmod 8)$ with $h(d)=1$.

Mollin and Williams Conjecture. Let $d=n^{2} \pm 2$ be a squarefree integer. Then $h(d)>1$ if $n>20$.

TheOREM 1.1. Let $d \not \equiv 5(\bmod 8)$ be a wide Richaud-Degert type. Then $h(d)=1$ if and only if

$$
d=3,6,7,11,14,23,33,38,47,62,83,167,227,398 .
$$

2. Computation of special values of the zeta function associated with $\mathbb{Q}\left(\sqrt{n^{2}-2}\right)$. Let $d=n^{2}-2$ be a positive square free integer, $K=$ $\mathbb{Q}(\sqrt{d})$ and $O(K)$ the ring of integers of $K$. Then $\epsilon=n^{2}-1+n \sqrt{n^{2}-2}$ is a fundamental unit of $K$ (see [8]), and $\{1, \omega\}$ is an integral basis for $O(K)$, where $\omega=\sqrt{n^{2}-2}$. For an integral ideal $\mathbf{a}$, let $N(\mathbf{a})$ be the number of 
cosets of $O(K) / \mathbf{a}$, and for an element $\alpha$ of $K$, let $N_{K}(\alpha)=\alpha \cdot \bar{\alpha}$. We note that $N_{K}(\epsilon)=1$. Let $I(K)$ be the set of nonzero fractional ideals of $K$. Let $K^{+}$be the set of totally positive elements in $K$, and $i\left(K^{+}\right)$be the set of principal fractional ideals generated by the elements in $K^{+}$. Let $\chi$ be an odd primitive character with conductor $q$. Then from the fact that $N_{K}(\epsilon)=1$ and $N_{K}(\omega)<0$, we have the following proposition.

Proposition 2.1 ([9, pp. 242-243]). If $h(d)=1$, then

$$
I(K)=(q) \cdot i\left(K^{+}\right) \cup(q \omega) \cdot i\left(K^{+}\right) .
$$

Thus if $h(d)=1$, then

$$
\begin{aligned}
\zeta_{K}(s, \chi) & :=\sum_{\substack{\mathbf{a} \in I(K) \\
\text { integral }}} \frac{\chi(N(\mathbf{a}))}{N(\mathbf{a})^{s}} \\
& =\sum_{\substack{\mathbf{a} \in(q) \cdot i\left(K^{+}\right) \\
\text {integral }}} \frac{\chi(N(\mathbf{a}))}{N(\mathbf{a})^{s}}+\sum_{\substack{\mathbf{a} \in(q \omega) \cdot i\left(K^{+}\right) \\
\text {integral }}} \frac{\chi(N(\mathbf{a}))}{N(\mathbf{a})^{s}} .
\end{aligned}
$$

By defining $R(\mathbf{b}):=\{a+b \epsilon \mid a, b \in \mathbb{Q}$ with $0<a \leq 1,0 \leq b<1$ and $\mathbf{b} \cdot(a+b \epsilon) \subset O(K)\}$ for an integral ideal $\mathbf{b}$ in $K$, we have the following propositon.

Proposition 2.2 ([5, Lemma 2.2]). An integral ideal a of $K$ is in $\mathbf{b} \cdot i\left(K^{+}\right):=\left\{\mathbf{b} \cdot \mathbf{c} \mid \mathbf{c} \in i\left(K^{+}\right)\right\}$if and only if

$$
\mathbf{a}=\mathbf{b} \cdot\left(a+b \epsilon+n_{1}+n_{2} \epsilon\right)
$$

for some $a+b \epsilon \in R(\mathbf{b})$ and nonnegative integers $n_{1}, n_{2}$.

In the following lemma, we find the set of all $(x, y)$ for which $x+y \epsilon \in$ $R((q))$.

LEMMA 2.3.

$$
\begin{aligned}
& \{(x, y) \mid x+y \epsilon \in R((q))\} \\
& =\left\{(x, y) \mid x=-\frac{r_{C, D}(n)}{q}+\frac{D+q j}{n q}+\sigma_{1}(j) \text { and } y=\frac{D+q j}{n q}\right. \\
& \quad \text { for } j=0,1, \ldots, n-1 \text { and } 0 \leq C, D \leq q-1\},
\end{aligned}
$$

where

$$
\sigma_{1}(j)= \begin{cases}1 & \text { if } 0 \leq j \leq\left[\frac{n r_{C, D}(n)-D}{q}\right], \\ 0 & \text { if }\left[\frac{n r_{C, D}(n)-D}{q}\right]+1 \leq j \leq n-1,\end{cases}
$$


and

$$
r_{C, D}(n)=n D-C-q\left[\frac{n D-C}{q}\right] .
$$

Proof. Since $\{C+D \omega \mid 0 \leq C, D \leq q-1\}$ represents all elements in $O(K) / q O(K)$, we have

$$
\begin{aligned}
& \{(x, y) \mid x+y \epsilon \in R((q))\} \\
& \quad=\left\{(x, y) \mid x+y \epsilon \in q^{-1} O(K) \text { and } 0<x \leq 1,0 \leq y<1\right\} \\
& =\{(x, y) \mid q(x+y \epsilon)=C+D \omega+q(i+j \omega) \text { for } 0 \leq C, D \leq q-1 \\
& \quad \text { integers } i, j \text { and } 0<x \leq 1,0 \leq y<1\} .
\end{aligned}
$$

Moreover, the equation

$$
\omega=\frac{\epsilon}{n}-\frac{n^{2}-1}{n}
$$

implies that

$$
q(x+y \epsilon)=C+q i+(D+q j)\left(\frac{\epsilon}{n}-\frac{n^{2}-1}{n}\right) .
$$

Hence

$$
\begin{aligned}
& y=\frac{D+q j}{n q}, \\
& x=\frac{C}{q}-\frac{(D+q j)\left(n^{2}-1\right)}{n q}+\left[1+\frac{(D+q j)\left(n^{2}-1\right)}{n q}-\frac{C}{q}\right]
\end{aligned}
$$

for $j=0,1, \ldots, n-1$. The equation

$$
\frac{(D+q j)\left(n^{2}-1\right)}{n q}-\frac{C}{q}=\left[\frac{n D-C}{q}\right]+\frac{r_{C, D}(n)}{q}-\frac{D+q j}{q n}+n j
$$

implies

$$
\begin{aligned}
\frac{C}{q}-\frac{(D+q j)\left(n^{2}-1\right)}{n q}+ & {\left[1+\frac{(D+q j)\left(n^{2}-1\right)}{n q}-\frac{C}{q}\right] } \\
=1 & -\frac{r_{C, D}(n)}{q}+\frac{D+q j}{q n}+\left[\frac{r_{C, D}(n)}{q}-\frac{D+q j}{q n}\right] .
\end{aligned}
$$

Since

$$
\left[\frac{r_{C, D}(n)}{q}-\frac{D+q j}{n q}\right]+1= \begin{cases}1 & \text { if } 0 \leq j \leq\left[\frac{n r_{C, D}(n)-D}{q}\right] \\ 0 & \text { if } 1+\left[\frac{n r_{C, D}(n)-D}{q}\right] \leq j \leq n-1\end{cases}
$$

the proof is complete. 
We also find the set of $(x, y)$ with $x+y \epsilon \in R((q \omega))$ :

Lemma 2.4.

$$
\begin{aligned}
& \{(x, y) \mid x+y \epsilon \in R((q \omega))\} \\
& =\left\{(x, y) \mid x=1+\frac{D}{q}-\frac{\left(n^{2}-1\right)(C+i q)}{q n\left(n^{2}-2\right)}+\sigma_{2}(i) \text { and } y=\frac{C+i q}{q n\left(n^{2}-2\right)}\right. \\
& \left.\quad \text { for } i=0,1, \ldots, n\left(n^{2}-2\right)-1 \text { and } 0 \leq C, D \leq q-1\right\},
\end{aligned}
$$

where

$$
\sigma_{2}(i)=k \Leftrightarrow l(k) \leq i<l(k+1)
$$

for $k=-1,0,1, \ldots, n^{2}-2$ and

$$
l(k)=\left\lceil\frac{n\left(n^{2}-2\right)(q k+D)}{q\left(n^{2}-1\right)}-\frac{C}{q}\right\rceil .
$$

Proof. The set $\{C+D \omega \mid 0 \leq C, D \leq q-1\}$ represents all elements in $O(K) / q O(K)$. So

$$
\begin{aligned}
& \{(x, y) \mid x+y \epsilon \in R((q \omega))\} \\
& =\left\{(x, y) \mid x+y \epsilon \in(q \omega)^{-1} O(K) \text { and } 0<x \leq 1,0 \leq y<1\right\} \\
& =\{(x, y) \mid q \omega(x+y \epsilon)=C+D \omega+q(i+j \omega) \text { for } 0 \leq C, D \leq q-1, \\
& \quad \text { integers } i, j \text { and } 0<x \leq 1,0 \leq y<1\} .
\end{aligned}
$$

From $\bar{\omega}=-\frac{\epsilon}{n}+\frac{n^{2}-1}{n}$, we deduce that

$$
\begin{aligned}
& q \omega(x+y \epsilon)=(C+q i)+\omega(D+q j) \Leftrightarrow \\
& \quad-q\left(n^{2}-2\right)(x+y \epsilon)=-\left(n^{2}-2\right)(D+q j)+\frac{n^{2}-1}{n}(C+i q)-\frac{C+i q}{n} \epsilon .
\end{aligned}
$$

So

$$
\begin{aligned}
& y=\frac{C+q i}{q n\left(n^{2}-2\right)}, \\
& x=1+\frac{D}{q}-\frac{\left(n^{2}-1\right)(C+i q)}{q n\left(n^{2}-2\right)}+\left[\frac{\left(n^{2}-1\right)(C+i q)}{q n\left(n^{2}-2\right)}-\frac{D}{q}\right]
\end{aligned}
$$

for $i=0,1, \ldots, n\left(n^{2}-2\right)-1$. By defining

$$
\sigma_{2}(i):=\left[\frac{\left(n^{2}-1\right)(C+i q)}{q n\left(n^{2}-2\right)}-\frac{D}{q}\right],
$$

we see that $\sigma_{2}(i)=k$ for $k=-1,0,1, \ldots, n^{2}-2$ if and only if

$$
\left\lceil\frac{n\left(n^{2}-2\right)(q k+D)}{q\left(n^{2}-1\right)}-\frac{C}{q}\right\rceil \leq i<\left\lceil\frac{n\left(n^{2}-2\right)(q(k+1)+D)}{q\left(n^{2}-1\right)}-\frac{C}{q}\right\rceil \text {. }
$$


From Lemmas 2.3 and 2.4, we deduce

Proposition 2.5. If $h\left(n^{2}-2\right)=1$, then

$$
\begin{aligned}
\zeta_{K}(0, \chi)= & \sum_{0 \leq C, D \leq q-1} \chi\left(C^{2}-\left(n^{2}-2\right) D^{2}\right) \\
& \times\left[\sum_{j=0}^{n-1} S\left(-\frac{r_{C, D}(n)}{q}+\frac{D+q j}{n q}+\sigma_{1}(j), \frac{D+q j}{n q}\right)\right. \\
& \left.-\sum_{i=0}^{\left(n^{2}-2\right) n-1} S\left(1-\frac{(C+i q)\left(n^{2}-1\right)}{q n\left(n^{2}-2\right)}+\frac{D}{q}+\sigma_{2}(i), \frac{C+i q}{q n\left(n^{2}-2\right)}\right)\right]
\end{aligned}
$$

where $S(x, y)=B_{1}(x) B_{1}(y)+\frac{1}{4}(\epsilon+\bar{\epsilon})\left(B_{2}(x)+B_{2}(y)\right)$ and $B_{1}, B_{2}$ are the first and second Bernoulli polynomials.

Proof. By Proposition 2.2, we have

$$
\begin{gathered}
\sum_{\substack{\mathbf{a} \in(q) \cdot i\left(K^{+}\right) \\
\text {integral }}} \frac{\chi(N(\mathbf{a}))}{N(\mathbf{a})^{s}}=\sum_{x+y \epsilon \in R((q))} \sum_{n_{1}, n_{2}=0}^{\infty} \frac{\chi\left(N\left((q) \cdot\left(x+y \epsilon+n_{1}+n_{2} \epsilon\right)\right)\right)}{N\left((q) \cdot\left(x+y \epsilon+n_{1}+n_{2} \epsilon\right)\right)^{s}} \\
\quad=\sum_{x+y \epsilon \in R((q))} \chi\left(N_{K}(q(x+y \epsilon))\right) \sum_{n_{1}, n_{2}=0}^{\infty} N_{K}\left(q\left(x+y \epsilon+n_{1}+n_{2} \epsilon\right)\right)^{-s}
\end{gathered}
$$

and

$$
\begin{aligned}
& \sum_{\substack{\mathbf{a} \in(q \omega) \cdot i\left(K^{+}\right) \\
\text {integral }}} \frac{\chi(N(\mathbf{a}))}{N(\mathbf{a})^{s}} \\
= & \sum_{x+y \epsilon \in R((q \omega))} \sum_{n_{1}, n_{2}=0}^{\infty} \frac{\chi\left(N\left((q \omega) \cdot\left(x+y \epsilon+n_{1}+n_{2} \epsilon\right)\right)\right)}{N\left((q \omega) \cdot\left(x+y \epsilon+n_{1}+n_{2} \epsilon\right)\right)^{s}} \\
= & \sum_{x+y \epsilon \in R((q \omega))} \chi\left(-N_{K}(q \omega(x+y \epsilon))\right) \sum_{n_{1}, n_{2}=0}^{\infty}\left(-N_{K}\left(q \omega\left(x+y \epsilon+n_{1}+n_{2} \epsilon\right)\right)\right)^{-s} .
\end{aligned}
$$

Now we recall Shintani's result in [13], [14]:

$$
\left.\sum_{n_{1}, n_{2}=0}^{\infty} N_{K}\left(x+y \epsilon+n_{1}+n_{2} \epsilon\right)^{-s}\right|_{s=0}=S(x, y) .
$$

We note that for $x+y \epsilon \in R((q))$,

$$
\begin{aligned}
N_{K}(q(x+y \epsilon)) & =N_{K}(C+D \omega+q(i+j \omega)) \\
& \equiv N_{K}(C+D \omega)(\bmod q)=C^{2}-\left(n^{2}-2\right) D^{2},
\end{aligned}
$$


and for $x+y \epsilon \in R((q \omega))$,

$$
\begin{aligned}
N_{K}(q \omega(x+y \epsilon)) & =N_{K}(C+D \omega+q(i+j \omega)) \\
& \equiv N_{K}(C+D \omega)(\bmod q)=C^{2}-\left(n^{2}-2\right) D^{2} .
\end{aligned}
$$

From Lemmas 2.3, 2.4 and the equations (1)-(6), we deduce the assertion immediately.

We observe that $-1 \leq \sigma_{2}(i) \leq n^{2}-2$ for $0 \leq i \leq n\left(n^{2}-2\right)-1$ and $\sigma_{2}(i)=k \Leftrightarrow l(k) \leq i<l(k+1)$. So we have

$$
\begin{aligned}
\sum_{i=0}^{n\left(n^{2}-2\right)-1} \sigma_{2}(i) & =\sum_{i=0}^{l(0)-1}(-1)+\sum_{k=0}^{n^{2}-3} \sum_{i=l(k)}^{l(k+1)-1} k+\left(n^{2}-2\right) \sum_{i=l\left(n^{2}-2\right)}^{n\left(n^{2}-2\right)-1} 1, \\
\sum_{i=0}^{n\left(n^{2}-2\right)-1} \sigma_{2}(i)^{2} & =\sum_{i=0}^{l(0)-1} 1+\sum_{k=0}^{n^{2}-3 l(k+1)-1} \sum_{i=l(k)}^{n\left(n^{2}-2\right)-1} k^{2}+\left(n^{2}-2\right)^{2} \sum_{i=l\left(n^{2}-2\right)}^{n\left(n^{2}-2\right)-1} 1, \\
\sum_{i=0}^{n\left(n^{2}-2\right)-1} \sigma_{2}(i) i & =\sum_{i=0}^{l(0)-1}(-i)+\sum_{k=0}^{n^{2}-3} \sum_{i=l(k)}^{l(k+1)-1} k i+\left(n^{2}-2\right) \sum_{i=l\left(n^{2}-2\right)} i,
\end{aligned}
$$

where $l(k)$ is computed in the following lemma.

Lemma 2.6. Let

$$
\begin{aligned}
& s_{C, D}(n)=r_{C, D}(n) n-D-q\left[\frac{r_{C, D}(n) n-D}{q}\right], \quad u_{C, D}(n)=\left[\frac{n D-C}{q}\right], \\
& v_{C, D}(n)=\left[\frac{r_{C, D}(n) n-D}{q}\right], \quad w_{C, D}(n)=\left\lceil\frac{s_{C, D}(n) n-r_{C, D}(n)}{q}\right\rceil .
\end{aligned}
$$

Assume $n>q$ and $0 \leq k \leq n^{2}-2$ with $k=n k_{1}+k_{2}\left(k_{2}=0,1, \ldots, n-1\right)$. If $r_{C, D}(n) \neq 0$ or $s_{C, D}(n)=0$, then

$$
l(k)= \begin{cases}u_{C, D}(n)+n k-k_{1}+1 & \text { if } k_{1}<w_{C, D}(n) \text { and } k_{2} \leq v_{C, D}(n), \\ u_{C, D}(n)+n k-k_{1} & \text { if } k_{1}<w_{C, D}(n) \text { and } k_{2}>v_{C, D}(n), \\ u_{C, D}(n)+n k-k_{1}+1 & \text { if } k_{1} \geq w_{C, D}(n) \text { and } k_{2}<v_{C, D}(n), \\ u_{C, D}(n)+n k-k_{1} & \text { if } k_{1} \geq w_{C, D}(n) \text { and } k_{2} \geq v_{C, D}(n),\end{cases}
$$

while if $r_{C, D}(n)=0$ and $s_{C, D}(n) \neq 0$, then

$$
l(k)= \begin{cases}u_{C, D}(n)+n k-k_{1}-1 & \text { if } k_{1} \geq w_{C, D}(n)-1 \text { and } k_{2}=n-1, \\ u_{C, D}(n)+n k-k_{1} & \text { otherwise. }\end{cases}
$$


Proof. Note that

$$
\begin{aligned}
l(k) & =\left\lceil\frac{n\left(n^{2}-2\right)(q k+D)}{q\left(n^{2}-1\right)}-\frac{C}{q}\right\rceil \\
& =n k+\left\lceil\frac{n D-C}{q}-\frac{k}{n-\frac{1}{n}}-\frac{D}{q\left(n-\frac{1}{n}\right)}\right\rceil .
\end{aligned}
$$

Moreover,

$$
\begin{aligned}
& \left\lceil\frac{n D-C}{q}-\frac{k}{n-\frac{1}{n}}-\frac{D}{q\left(n-\frac{1}{n}\right)}\right\rceil \\
& =\left\lceil\frac{n D-C}{q}\right]+\left\lceil\frac{r_{C, D}(n)}{q}-\frac{\frac{D}{q}+\frac{k_{1}}{n}+k_{2}}{n-\frac{1}{n}}\right\rceil-k_{1} .
\end{aligned}
$$

Now we observe that

$$
\begin{aligned}
&\left\lceil\frac{r_{C, D}(n)}{q}-\frac{\frac{D}{q}+}{n}+\frac{k_{1}}{n}+k_{2}\right. \\
&\left.n-\frac{1}{n}\right\rceil \\
&= {\left[\frac{\left[\frac{r_{C, D}(n) n-D}{q}\right]-k_{2}}{n-\frac{1}{n}}+\frac{\left[\frac{s_{C, D}(n) n-r_{C, D}(n)}{q}\right]-k_{1}}{n\left(n-\frac{1}{n}\right)}\right.} \\
&\left.+\frac{s_{C, D}(n) n-r_{C, D}(n)-q\left[\frac{s_{C, D}(n) n-r_{C, D}(n)}{q}\right]}{q n\left(n-\frac{1}{n}\right)}\right\rceil .
\end{aligned}
$$

We consider the case of $r_{C, D}(n) \neq 0$ or $s_{C, D}(n)=0$,

$$
k_{1}<\left\lceil\frac{s_{C, D}(n) n-r_{C, D}(n)}{q}\right\rceil \text { and } \quad k_{2} \leq\left[\frac{r_{C, D}(n) n-D}{q}\right] \text {. }
$$

For $n>q$ we have $\frac{s_{C, D}(n) n-r_{C, D}(n)}{q}<n-1$ and $\frac{r_{C, D}(n) n-D}{q}<n-1$. So

$$
\left[\frac{s_{C, D}(n) n-r_{C, D}(n)}{q}\right] \leq n-2 \text { and }\left[\frac{r_{C, D}(n) n-D}{q}\right] \leq n-2 .
$$

If $s_{C, D}(n) n-r_{C, D}(n)-q\left[\frac{s_{C, D}(n) n-r_{C, D}(n)}{q}\right]=0$, then $\left\lceil\frac{s_{C, D}(n) n-r_{C, D}(n)}{q}\right\rceil=$ $\left[\frac{s_{C, D}(n) n-r_{C, D}(n)}{q}\right]$. Thus from (11), (12), we have

$$
\begin{aligned}
& 1 \leq\left[\frac{s_{C, D}(n) n-r_{C, D}(n)}{q}\right]-k_{1} \leq n-2, \\
& 0 \leq\left[\frac{r_{C, D}(n) n-D}{q}\right]-k_{2} \leq n-2 .
\end{aligned}
$$

Hence

$$
\frac{1}{n\left(n-\frac{1}{n}\right)} \leq \frac{\left[\frac{r_{C, D}(n) n-D}{q}\right]-k_{2}}{n-\frac{1}{n}}+\frac{\left[\frac{s_{C, D}(n) n-r_{C, D}(n)}{q}\right]-k_{1}}{n\left(n-\frac{1}{n}\right)} \leq \frac{n^{2}-n-2}{n^{2}-1}<1 .
$$


So

$$
\left\lceil\frac{\left[\frac{r_{C, D}(n) n-D}{q}\right]-k_{2}}{n-\frac{1}{n}}+\frac{\left[\frac{s_{C, D}(n) n-r_{C, D}(n)}{q}\right]-k_{1}}{n\left(n-\frac{1}{n}\right)}\right\rceil=1 .
$$

If $s_{C, D}(n) n-r_{C, D}(n)-q\left[\frac{s_{C, D}(n) n-r_{C, D}(n)}{q}\right] \neq 0$, then $\left\lceil\frac{s_{C, D}(n) n-r_{C, D}(n)}{q}\right\rceil=$ $\left[\frac{s_{C, D}(n) n-r_{C, D}(n)}{q}\right]+1$. From (11), (12), we have

$$
\begin{aligned}
& 0 \leq\left[\frac{s_{C, D}(n) n-r_{C, D}(n)}{q}\right]-k_{1} \leq n-2, \\
& 0 \leq\left[\frac{r_{C, D}(n) n-D}{q}\right]-k_{2} \leq n-2,
\end{aligned}
$$

and

$$
1 \leq s_{C, D}(n) n-r_{C, D}(n)-q\left[\frac{s_{C, D}(n) n-r_{C, D}(n)}{q}\right] \leq q-1 .
$$

Thus

$$
\begin{aligned}
0< & \frac{1}{q n\left(n-\frac{1}{n}\right)} \leq \frac{\left[\frac{r_{C, D}(n) n-D}{q}\right]-k_{2}}{n-\frac{1}{n}}+\frac{\left[\frac{s_{C, D}(n) n-r_{C, D}(n)}{q}\right]-k_{1}}{n\left(n-\frac{1}{n}\right)} \\
& +\frac{s_{C, D}(n) n-r_{C, D}(n)-q\left[\frac{s_{C, D}(n) n-r_{C, D}(n)}{q}\right]}{q n\left(n-\frac{1}{n}\right)} \\
\leq & \frac{n^{2}-n-2}{n^{2}-1}+\frac{q-1}{q n\left(n-\frac{1}{n}\right)}<1 .
\end{aligned}
$$

So

$$
\begin{aligned}
\left\lceil\frac{\left[\frac{r_{C, D}(n) n-D}{q}\right]-}{n-\frac{1}{n}}+\frac{\left[\frac{s_{C, D}(n) n-r_{C, D}(n)}{q}\right]-k_{1}}{n\left(n-\frac{1}{n}\right)}\right. \\
\left.\quad+\frac{s_{C, D}(n) n-r_{C, D}(n)-q\left[\frac{s_{C, D}(n) n-r_{C, D}(n)}{q}\right]}{q n\left(n-\frac{1}{n}\right)}\right]=1 .
\end{aligned}
$$

From (8)-(10) and (13)-(14), we have

$$
l(k)=\left[\frac{n D-C}{q}\right]+n k-k_{1}+1 .
$$

The other cases can be handled similarly.

We can express the summation (7) more explicitly using Lemma 2.6. In case $r_{C, D}(n) \neq 0$ or $s_{C, D}(n)=0$, we have 


$$
\begin{aligned}
& \sum_{k=0}^{n^{2}-3} \sum_{i=l(k)}^{l(k+1)-1} \\
& =\sum_{k_{1}=0}^{w-1}\left(\sum_{k_{2}=0}^{v-1} \sum_{i=n\left(k_{1} n+k_{2}\right)+u-k_{1}+1}^{n\left(k_{1} n+k_{2}+1\right)+u-k_{1}}+\sum_{i=n\left(k_{1} n+v\right)+u-k_{1}+1}^{n\left(k_{1} n+v+1\right)+u-k_{1}-1}+\sum_{k_{2}=v+1}^{n-1} \sum_{i=n\left(k_{1} n+k_{2}\right)+u-k_{1}}^{n\left(k_{1} n+k_{2}+1\right)+u-k_{1}-1}\right) \\
& +\sum_{k_{1}=w}^{n-2}\left(\sum_{k_{2}=0}^{v-2} \sum_{i=n\left(k_{1} n+k_{2}\right)+u-k_{1}+1}^{n\left(k_{1} n+k_{2}+1\right)+u-k_{1}}+\sum_{i=n\left(k_{1} n+v-1\right)+u-k_{1}+1}^{n\left(k_{1} n+v\right)+u-k_{1}-1}+\sum_{k_{2}=v}^{n-1} \sum_{i=n\left(k_{1} n+k_{2}\right)+u-k_{1}}^{n\left(k_{1} n+k_{2}+1\right)+u-k_{1}-1}\right) \\
& +\sum_{k_{2}=0}^{v-2} \sum_{i=n\left(n^{2}-n+k_{2}\right)+u-n+2}^{n\left(n^{2}-n+k_{2}+1\right)+u-n+1}+\sum_{i=n\left(n^{2}-n+v-1\right)+u-n+2}^{n\left(n^{2}-n+v\right)+u-n}+\sum_{k_{2}=v}^{n-3} \sum_{i=n\left(n^{2}-n+k_{2}\right)+u-n+1}^{n\left(n^{2}-n+k_{2}+1\right)+u-n},
\end{aligned}
$$

while in case $r_{C, D}(n)=0$ and $s_{C, D}(n) \neq 0$, we have

$$
\begin{aligned}
& \sum_{k=0}^{n^{2}-3} \sum_{i=l(k)}^{l(k+1)-1} \\
& =\sum_{k_{1}=0}^{\omega-2}\left(\sum_{k_{2}=0}^{n-2} \sum_{n\left(k_{1} n+k_{2}\right)+u-k_{1}}^{n\left(k_{1} n+k_{2}+1\right)+u-k_{1}-1}+\sum_{n\left(k_{1} n+n-1\right)+u-k_{1}}^{n\left(k_{1} n+n\right)+u-k_{1}-2}\right) \\
& +\sum_{k_{1}=\omega-1}^{n-2}\left(\sum_{k_{2}=0}^{n-3} \sum_{n\left(k_{1} n+k_{2}\right)+u-k_{1}}^{n\left(k_{1} n+k_{2}+1\right)+u-k_{1}-1}+\sum_{n\left(k_{1} n+n-2\right)+u-k_{1}}^{n\left(k_{1} n+n-1\right)+u-k_{1}-2}+\sum_{n\left(k_{1} n+n-1\right)+u-k_{1}-1}^{n\left(k_{1} n+n\right)+u-k_{1}-2}\right) \\
& +\sum_{k_{2}=0}^{n-3} \sum_{n\left(n^{2}-n+k_{2}\right)+u-n+1}^{n\left(n^{2}-n+k_{2}+1\right)+u-n} \text {. }
\end{aligned}
$$

(In the above two equations, $u, v, w$ are $u_{C, D}(n), v_{C, D}(n), w_{C, D}(n)$ respectively.) From this expression of $l(k)$, we can calculate $\sum \sigma_{2}(i), \sum i \sigma_{2}(i)$ and $\sum \sigma_{2}(i)^{2}$ by computer. If $r_{C, D}(n) \neq 0$ or $s_{C, D}(n)=0$ then

$$
\begin{aligned}
& n\left(n^{2}-2\right)-1 \\
& \sum_{i=0} \sigma_{2}(i)=\frac{1}{2}\left[-4 n^{3}+n^{5}-n^{2}\left(1+2 u_{C, D}(n)\right)\right. \\
& \left.-2 n\left(-2+v_{C, D}(n)\right)+2\left(1+u_{C, D}(n)-w_{C, D}(n)\right)\right], \\
& n\left(n^{2}-2\right)-1 \\
& \sum_{i=0} \sigma_{2}(i)^{2}= \\
& -3-\frac{11 n^{5}}{6}+\frac{n^{7}}{3}-3 u_{C, D}(n)-\frac{1}{2} n^{4}\left(1+2 u_{C, D}(n)\right)-n^{3}\left(-3+v_{C, D}(n)\right) \\
& +n^{2}\left(3+4 u_{C, D}(n)+v_{C, D}(n)\right)+w_{C, D}(n)-2 v_{C, D}(n) w_{C, D}(n) \\
& -n\left(2-2 v_{C, D}(n)+v_{C, D}(n)^{2}-w_{C, D}(n)+w_{C, D}(n)^{2}\right) \text {, }
\end{aligned}
$$




$$
\begin{aligned}
& \quad \sum_{i=0}^{n\left(n^{2}-2\right)-1} i \sigma_{2}(i)= \\
& \frac{1}{12}\left[-23 n^{6}+4 n^{8}-6 n^{5}\left(1+u_{C, D}(n)\right)+n^{4}\left(41-6 v_{C, D}(n)\right)\right. \\
& +6 n^{3}\left(5+4 u_{C, D}(n)+v_{C, D}(n)\right) \\
& -6 n\left(6+v_{C, D}(n)+2 u_{C, D}(n)\left(2+v_{C, D}(n)\right)+2 v_{C, D}(n) w_{C, D}(n)\right) \\
& +6\left(u_{C, D}(n)+u_{C, D}(n)^{2}-2 u_{C, D}(n) w_{C, D}(n)+\left(-1+w_{C, D}(n)\right) w_{C, D}(n)\right) \\
& \left.-2 n^{2}\left(11+3 u_{C, D}(n)^{2}-6 v_{C, D}(n)+3 v_{C, D}(n)^{2}-3 w_{C, D}(n)+3 w_{C, D}(n)^{2}\right)\right] .
\end{aligned}
$$

If $r_{C, D}(n)=0$ and $s_{C, D}(n) \neq 0$ then

$$
\begin{aligned}
& \sum_{i=0}^{n\left(n^{2}-2\right)-1} \sigma_{2}(i)=1+3 n-2 n^{3}+\frac{n^{5}}{2}-w_{C, D}(n)+u_{C, D}(n) \\
& \quad-\frac{1}{2} n^{2}\left(1+2 u_{C, D}(n)\right), \\
& \begin{array}{l}
n\left(n^{2}-2\right)-1 \\
\sum_{i=0} \sigma_{2}(i)^{2}=
\end{array} \\
& \quad 4 n^{3}-\frac{11 n^{5}}{6}+\frac{n^{7}}{3}-\frac{n^{4}}{2}\left(1+2 u_{C, D}(n)\right)+n^{2}\left(2+4 u_{C, D}(n)\right) \\
& \quad-3\left(1+u_{C, D}(n)-w_{C, D}(n)\right)+n\left(-5+w_{C, D}(n)-w_{C, D}(n)^{2}\right), \\
& \quad \sum_{i=0}^{n\left(n^{2}-2\right)-1} i \sigma_{2}(i)= \\
& \quad \frac{1}{12}\left[47 n^{4}-23 n^{6}+4 n^{8}+24 n^{3}\left(1+u_{C, D}(n)\right)-6 n^{5}\left(1+u_{C, D}(n)\right)\right. \\
& \quad-6 n\left(5+2 u_{C, D}(n)-2 w_{C, D}(n)\right) \\
& \quad+6\left(u_{C, D}(n)+u_{C, D}(n)^{2}-2 u_{C, D}(n) w_{C, D}(n)+\left(-1+w_{C, D}(n)\right) w_{C, D}(n)\right) \\
& \left.\quad-2 n^{2}\left(20+3 u_{C, D}(n)^{2}-3 w_{C, D}(n)+3 w_{C, D}(n)^{2}\right)\right] .
\end{aligned}
$$

We will use these expressions in the proof of (ii) of the next proposition.

Proposition 2.7. If $n>q$ then

$$
\text { (i) } \begin{aligned}
& \sum_{j=0}^{n-1} S\left(-\frac{r_{C, D}(n)}{q}+\frac{D+q j}{n q}+\sigma_{1}(j), \frac{D+q j}{n q}\right) \\
= & -\frac{1}{2}+\frac{n}{4}+\frac{D^{2} n}{2 q^{2}}+\frac{r_{C, D}(n)}{q}-\frac{n r_{C, D}(n)^{2}}{2 q^{2}}-\frac{v_{C, D}(n)}{2}+\frac{r_{C, D}(n) v_{C, D}(n)}{q} \\
& -\frac{n D}{2 q}+\frac{n s_{C, D}(n)^{2}}{2 q^{2}}-\frac{n s_{C, D}(n)}{2 q}
\end{aligned}
$$




$$
\text { (ii) } \begin{aligned}
\quad \sum_{i=0}^{\left(n^{2}-2\right) n-1} S\left(1-\frac{(C+i q)\left(n^{2}-1\right)}{q n\left(n^{2}-2\right)}+\frac{D}{q}+\sigma_{2}(i), \frac{C+i q}{q n\left(n^{2}-2\right)}\right) \\
=\frac{1}{2}-\frac{n}{4}-\frac{n r_{C, D}(n) C}{q^{2}}+\frac{n r_{C, D}(n)}{2 q}-\frac{3 n r_{C, D}(n)^{2}}{2 q^{2}}+\frac{u_{C, D}(n)}{2}+\frac{C v_{C, D}(n)}{q} \\
+\frac{r_{C, D}(n) v_{C, D}(n)}{q}+\frac{w_{C, D}(n)}{2}-\frac{D}{q}+\frac{3 n D^{2}}{2 q^{2}}-\frac{2 u_{C, D}(n) D}{q}+\frac{n s_{C, D}(n)^{2}}{2 q^{2}} \\
-\frac{u_{C, D}(n) s_{C, D}(n)}{q}-\frac{w_{C, D}(n) s_{C, D}(n)}{q}+\frac{n s_{C, D}(n) D}{q^{2}}
\end{aligned}
$$

where $r_{C, D}(n), s_{C, D}(n), u_{C, D}(n), v_{C, D}(n), w_{C, D}(n)$ are as in Lemma 2.6.

Proof. (i) By computer, we obtain the following equation:

$$
\begin{aligned}
& \sum_{j=0}^{n-1} S\left(-\frac{r_{C, D}(n)}{q}+\frac{D+q j}{n q}+\sigma_{1}(j), \frac{D+q j}{n q}\right) \\
& =\frac{n D^{2}}{q^{2}}-\frac{n^{2} r_{C, D}(n) D}{q^{2}}+\frac{n^{3} r_{C, D}(n)^{2}}{2 q^{2}}+\frac{n v_{C, D}(n) D}{q}-\frac{n^{2} r_{C, D}(n) v_{C, D}(n)}{q} \\
& \quad+\frac{n v_{C, D}(n)^{2}}{2}-\frac{n^{2} r_{C, D}(n)}{2 q}+\frac{n v_{C, D}(n)}{2}-\frac{1}{2}+\frac{n}{4}+\frac{r_{C, D}(n)}{q}-\frac{n r_{C, D}(n)^{2}}{2 q^{2}} \\
& \quad-\frac{v_{C, D}(n)}{2}+\frac{r_{C, D}(n) v_{C, D}(n)}{q} .
\end{aligned}
$$

From the equation

$$
s_{C, D}(n)=n r_{C, D}(n)-D-q\left[\frac{n r_{C, D}(n)-D}{q}\right]=n r_{C, D}(n)-D-q v_{C, D}(n)
$$

we have

$$
\begin{aligned}
\frac{n D^{2}}{2 q^{2}}-\frac{n^{2} r_{C, D}(n) D}{q^{2}}+ & \frac{n^{3} r_{C, D}(n)^{2}}{2 q^{2}}+\frac{n v_{C, D}(n) D}{q} \\
& -\frac{n^{2} r_{C, D}(n) v_{C, D}(n)}{q}+\frac{n v_{C, D}(n)^{2}}{2} \\
= & \frac{n\left(n r_{C, D}(n)-D-q v_{C, D}(n)\right)^{2}}{2 q^{2}}=\frac{n s_{C, D}(n)^{2}}{2 q^{2}}
\end{aligned}
$$

and

$$
\begin{aligned}
-\frac{n^{2} r_{C, D}(n)}{2 q}+\frac{n v_{C, D}(n)}{2} & =\frac{-n\left(n r_{C, D}(n)-D-q v_{C, D}(n)\right)}{2 q}-\frac{n D}{2 q} \\
& =-\frac{n s_{C, D}(n)}{2 q}-\frac{n D}{2 q}
\end{aligned}
$$

Hence we can obtain (i) directly. 
(ii) From our expressions above for the sums $\sum_{i=0}^{n\left(n^{2}-2\right)-1} \sigma_{2}(i)$, $\sum_{i=0}^{n\left(n^{2}-2\right)-1} \sigma_{2}(i)^{2}$ and $\sum_{i=0}^{n\left(n^{2}-2\right)-1} \sigma_{2}(i) i$, we can also calculate

$$
\sum_{i=0}^{\left(n^{2}-2\right) n-1} S\left(1-\frac{(C+i q)\left(n^{2}-1\right)}{q n\left(n^{2}-2\right)}+\frac{D}{q}+\sigma_{2}(i), \frac{C+i q}{q n\left(n^{2}-2\right)}\right) .
$$

Here are the results. In case $r_{C, D}(n) \neq 0$ or $s_{C, D}(n)=0$, we have

$$
\sum_{i=0}^{\left(n^{2}-2\right) n-1} S\left(1-\frac{(C+i q)\left(n^{2}-1\right)}{q n\left(n^{2}-2\right)}+\frac{D}{q}+\sigma_{2}(i), \frac{C+i q}{q n\left(n^{2}-2\right)}\right)
$$$$
=\frac{1}{2}-\frac{n}{4}+\frac{C^{2}}{n q^{2}}-\frac{C}{n q}+\frac{2 C r_{C, D}(n)}{n q^{2}}-\frac{C n r_{C, D}(n)}{q^{2}}-\frac{r_{C, D}(n)}{n q}+\frac{n r_{C, D}(n)}{2 q}
$$$$
+\frac{r_{C, D}(n)^{2}}{n q^{2}}-\frac{3 n r_{C, D}(n)^{2}}{2 q^{2}}+\frac{n^{3} r_{C, D}(n)^{2}}{2 q^{2}}+\frac{u_{C, D}(n)}{2}-\frac{u_{C, D}(n)}{n}
$$$$
+\frac{C u_{C, D}(n)}{n q}+\frac{r_{C, D}(n) u_{C, D}(n)}{n q}-\frac{n r_{C, D}(n) u_{C, D}(n)}{q}+\frac{C v_{C, D}(n)}{q}
$$$$
+\frac{r_{C, D}(n) v_{C, D}(n)}{q}-\frac{n^{2} r_{C, D}(n) v_{C, D}(n)}{q}+u_{C, D}(n) v_{C, D}(n)+\frac{n v_{C, D}(n)^{2}}{2}
$$$$
+\frac{w_{C, D}(n)}{2}+\frac{C w_{C, D}(n)}{n q}+\frac{r_{C, D}(n) w_{C, D}(n)}{n q}-\frac{n r_{C, D}(n) w_{C, D}(n)}{q}
$$$$
+\frac{u_{C, D}(n) w_{C, D}(n)}{q}+v_{C, D}(n) w_{C, D}(n)
$$$$
=\left(\frac{C^{2}}{n q^{2}}+\frac{2 C r_{C, D}(n)}{n q^{2}}+\frac{r_{C, D}(n)^{2}}{n q^{2}}+\frac{2 C u_{C, D}(n)}{n q}+\frac{2 r_{C, D}(n) u_{C, D}(n)}{n q}\right)
$$$$
+\left(-\frac{C}{n q}-\frac{r_{C, D}(n)}{n q}-\frac{u_{C, D}(n)}{n}\right)
$$$$
+\left(\frac{C w_{C, D}(n)}{n q}+\frac{r_{C, D}(n) w_{C, D}(n)}{n q}+\frac{u_{C, D}(n) w_{C, D}(n)}{n}\right)
$$$$
+\left(-\frac{C u_{C, D}(n)}{n q}-\frac{r_{C, D}(n) u_{C, D}(n)}{n q}\right)
$$$$
+\left(-\frac{n r_{C, D}(n) w_{C, D}(n)}{q}+\frac{D w_{C, D}(n)}{q}+v_{C, D}(n) w_{C, D}(n)\right)
$$$$
+\left(\frac{n^{3} r_{C, D}(n)^{2}}{2 q^{2}}-\frac{n^{2} r_{C, D}(n) v_{C, D}(n)}{q}+\frac{n v_{C, D}(n)^{2}}{2}+\frac{n D^{2}}{2 q^{2}}\right)
$$$$
+\left(-\frac{n r_{C, D}(n) u_{C, D}(n)}{q}+\frac{D u_{C, D}(n)}{q}+u_{C, D}(n) v_{C, D}(n)\right)
$$ 


$$
\begin{aligned}
& -\frac{D w_{C, D}(n)}{q}-\frac{n D^{2}}{2 q^{2}}-\frac{D u_{C, D}(n)}{q}-\frac{C n r_{C, D}(n)}{q^{2}}+\frac{n r_{C, D}(n)}{2 q} \\
& -\frac{3 n r_{C, D}(n)^{2}}{2 q^{2}}+\frac{u_{C, D}(n)}{2}+\frac{C v_{C, D}(n)}{q}+\frac{r_{C, D}(n) v_{C, D}(n)}{q}+\frac{w_{C, D}(n)}{2} \\
& +\frac{1}{2}-\frac{n}{4} .
\end{aligned}
$$

In the case $r_{C, D}(n)=0$ and $s_{C, D}(n) \neq 0$, we have

$$
\begin{aligned}
\sum_{i=0}^{\left(n^{2}-2\right) n-1} S & \left(1-\frac{(C+i q)\left(n^{2}-1\right)}{q n\left(n^{2}-2\right)}+\frac{D}{q}+\sigma_{2}(i), \frac{C+i q}{q n\left(n^{2}-2\right)}\right) \\
= & \frac{1}{2}+\frac{n}{4}+\frac{C^{2}}{n q^{2}}-\frac{C}{n q}-\frac{u_{C, D}(n)}{2}-\frac{u_{C, D}(n)}{n}+\frac{C u_{C, D}(n)}{n q} \\
& -\frac{C}{q}-\frac{w_{C, D}(n)}{2}+\frac{C w_{C, D}(n)}{n q}+\frac{u_{C, D}(n) w_{C, D}(n)}{n} .
\end{aligned}
$$

Since $r_{C, D}(n)=0$ and $s_{C, D}(n) \neq 0$ implies that $v_{C, D}(n)=-1$, the equation (16) is obtained from $r_{C, D}(n)=0$ and $s_{C, D}(n) \neq 0$ and equation (15). Thus in either case we have (15). We note that $r_{C, D}(n)=n D-C-q u_{C, D}(n)$ implies that

$$
\begin{gathered}
\frac{C^{2}}{n q^{2}}+\frac{2 C r_{C, D}(n)}{n q^{2}}+\frac{r_{C, D}(n)^{2}}{n q^{2}}+\frac{2 C u_{C, D}(n)}{n q}+\frac{2 r_{C, D}(n) u_{C, D}(n)}{n q} \\
=\frac{\left(C+r_{C, D}(n)+q u_{C, D}(n)\right)^{2}}{n q^{2}}-\frac{u_{C, D}(n)^{2}}{n}=\frac{n D^{2}}{q^{2}}-\frac{u_{C, D}(n)^{2}}{n} \\
-\frac{C}{n q}-\frac{r_{C, D}(n)}{n q}-\frac{u_{C, D}(n)}{n}=-\frac{C+r_{C, D}(n)+q u_{C, D}(n)}{n q}=-\frac{D}{q} \\
\frac{C w_{C, D}(n)}{n q}+\frac{r_{C, D}(n) w_{C, D}(n)}{n q}+\frac{u_{C, D}(n) w_{C, D}(n)}{n} \\
=\frac{\left(C+r_{C, D}(n)+q u_{C, D}(n)\right) w_{C, D}(n)}{n q}=\frac{D w_{C, D}(n)}{q}
\end{gathered}
$$

and

$$
\begin{aligned}
-\frac{C u_{C, D}(n)}{n q} & -\frac{r_{C, D}(n) u_{C, D}(n)}{n q} \\
& =-\frac{\left(C+r_{C, D}(n)+u_{C, D}(n) q\right) u_{C, D}(n)}{n q}+\frac{u_{C, D}(n)^{2}}{n} \\
& =-\frac{D u_{C, D}(n)}{q}+\frac{u_{C, D}(n)^{2}}{n}
\end{aligned}
$$


Also the equation $s_{C, D}(n)=r_{C, D}(n) n-D-q v_{C, D}(n)$ implies that

$$
\begin{array}{r}
-\frac{n r_{C, D}(n) w_{C, D}(n)}{q}+\frac{D w_{C, D}(n)}{q}+v_{C, D}(n) w_{C, D}(n) \\
=-\frac{w_{C, D}(n)\left(r_{C, D}(n) n-D-q v_{C, D}(n)\right)}{q}=-\frac{w_{C, D}(n) s_{C, D}(n)}{q},
\end{array}
$$

$$
\begin{aligned}
& \frac{n^{3} r_{C, D}(n)^{2}}{2 q^{2}}-\frac{n^{2} r_{C, D}(n) v_{C, D}(n)}{q}+\frac{n v_{C, D}(n)^{2}}{2}+\frac{n D^{2}}{2 q^{2}} \\
& \quad=\frac{n\left(n r_{C, D}(n)-D-q v_{C, D}(n)\right)^{2}}{2 q^{2}}-\frac{n D v_{C, D}(n)}{q}+\frac{n^{2} r_{C, D}(n) D}{q^{2}} \\
& \quad=\frac{n s_{C, D}(n)^{2}}{2 q^{2}}+\frac{n D\left(r_{C, D}(n) n-D-q v_{C, D}(n)\right)}{q^{2}}+\frac{n D^{2}}{q^{2}} \\
& \quad=\frac{n s_{C, D}(n)^{2}}{2 q^{2}}+\frac{n D s_{C, D}(n)}{q^{2}}+\frac{n D^{2}}{q^{2}}
\end{aligned}
$$

and

$$
\begin{aligned}
& -\frac{n r_{C, D}(n) u_{C, D}(n)}{q}+\frac{D u_{C, D}(n)}{q}+u_{C, D}(n) v_{C, D}(n) \\
& =-\frac{u_{C, D}(n)\left(r_{C, D}(n) n-D-q v_{C, D}(n)\right)}{q}=-\frac{u_{C, D}(n) s_{C, D}(n)}{q} .
\end{aligned}
$$

From (17)-(23), we can deduce (ii).

Finally, we have the following theorem.

Theorem 2.8. If $h\left(n^{2}-2\right)=1$ and $n>q$, then

$$
\begin{aligned}
\zeta_{K}(0, \chi)= & \frac{1}{2 q^{2}} \sum_{0 \leq C, D \leq q-1} \chi\left(C^{2}-\left(n^{2}-2\right) D^{2}\right) \\
& \times\left[n\left(q^{2}-q s_{C, D}(n)+2 r_{C, D}(n)^{2}+2 D^{2}-2 q D-2 q r_{C, D}(n)\right)\right. \\
& +w_{C, D}(n)\left(-q^{2}+2 q s_{C, D}(n)\right)-2 q^{2}-4 D r_{C, D}(n)+q C \\
& \left.+q s_{C, D}(n)-2 s_{C, D}(n) r_{C, D}(n)+3 q r_{C, D}(n)+3 q D-2 C D\right],
\end{aligned}
$$

where $r_{C, D}(n), s_{C, D}(n), u_{C, D}(n), v_{C, D}(n), w_{C, D}(n)$ are as in Lemma 2.6.

Proof. By Propositions 2.5 and 2.7,

$$
\begin{aligned}
\zeta_{K}(0, \chi)= & \sum_{0 \leq C, D \leq q-1} \chi\left(C^{2}-\left(n^{2}-2\right) D^{2}\right) \\
& \times\left[\sum_{j=0}^{n-1} S\left(-\frac{r_{C, D}(n)}{q}+\frac{D+q j}{n q}+\sigma_{1}(j), \frac{D+q j}{n q}\right)\right.
\end{aligned}
$$




$$
\begin{aligned}
& \left.-\sum_{i=0}^{n\left(n^{2}-2\right)-1} S\left(1-\frac{(C+i q)\left(n^{2}-1\right)}{q n\left(n^{2}-2\right)}+\frac{D}{q}+\sigma_{2}(i), \frac{C+i q}{q n\left(n^{2}-2\right)}\right)\right] \\
= & \frac{1}{2 q^{2}} \sum_{0 \leq C, D \leq q-1} \chi\left(C^{2}-\left(n^{2}-2\right) D^{2}\right) \cdot\left[\left(-2 n D^{2}+4 q u_{C, D}(n) D\right)\right. \\
& +\left(-q n D-q^{2} u_{C, D}(n)\right)+\left(2 q u_{C, D}(n) s_{C, D}(n)-2 n D s_{C, D}(n)\right) \\
& +\left(-q^{2} v_{C, D}(n)-q n r_{C, D}(n)\right)+\left(2 C n r_{C, D}(n)-2 q C v_{C, D}(n)\right) \\
& -2 q^{2}+n q^{2}+2 q r_{C, D}(n)-q n s_{C, D}(n)-q^{2} w_{C, D}(n) \\
& \left.+2 q D+2 q w_{C, D}(n) s_{C, D}(n)+2 n r_{C, D}(n)^{2}\right] .
\end{aligned}
$$

The equation $n D-C-q u_{C, D}(n)=r_{C, D}(n)$ implies that

$$
\begin{aligned}
-2 n D^{2}+4 q u_{C, D}(n) D & =4 D\left(q u_{C, D}(n)-n D\right)+2 n D^{2} \\
& =-4 D r_{C, D}(n)-4 D C+2 n D^{2}, \\
-q n D-q^{2} u_{C, D}(n) & =q\left(n D-q u_{C, D}(n)\right)-2 q n D \\
& =q r_{C, D}(n)+q C-2 q n D
\end{aligned}
$$

and

(26) $2 q u_{C, D}(n) s_{C, D}(n)-2 n D s_{C, D}(n)=-2 s_{C, D}(n) r_{C, D}(n)-2 s_{C, D}(n) C$.

Also the equation $r_{C, D}(n) n-D-q v_{C, D}(n)=s_{C, D}(n)$ implies that

$$
\begin{aligned}
-q^{2} v_{C, D}(n)-q n r_{C, D}(n) & =-q\left(q v_{C, D}(n)-n r_{C, D}(n)\right)-2 q n r_{C, D}(n) \\
& =q D+q s_{C, D}(n)-2 q n r_{C, D}(n)
\end{aligned}
$$

and

$$
-2 q n v_{C, D}(n)+2 C n r_{C, D}(n)=2 C D+2 C s_{C, D}(n) .
$$

From (24)-(28), we can deduce the conclusion of the theorem.

Corollary 2.9. If $h\left(n^{2}-2\right)=1, n>q$ and $n=q k+r$ for $0 \leq r<q$ then $\zeta_{K}(0, \chi)=\frac{1}{2 q^{2}}\left(B_{\chi}(r) k+A_{\chi}(r)\right)$, where

$$
\begin{aligned}
A_{\chi}(r)= & \sum_{0 \leq C, D \leq q-1} \chi\left(C^{2}-\left(r^{2}-2\right) D^{2}\right) \\
& \times\left[r\left(q^{2}-q s_{C, D}(r)+2 r_{C, D}(r)^{2}+2 D^{2}-2 q D-2 q r_{C, D}(r)\right)\right. \\
& +w_{C, D}(r)\left(-q^{2}+2 q s_{C, D}(r)\right)-2 q^{2}-4 D r_{C, D}(r)+q C \\
& \left.-2 s_{C, D}(r) r_{C, D}(r)+q s_{C, D}(r)+3 q r_{C, D}(r)+3 q D-2 C D\right], \\
B_{\chi}(r)= & \sum_{0 \leq C, D \leq q-1} \chi\left(C^{2}-\left(r^{2}-2\right) D^{2}\right) \cdot\left[q^{3}-2 q^{2} s_{C, D}(r)+2 r_{C, D}(r)^{2} q\right. \\
& \left.+2 D^{2} q-2 D q^{2}-2 q^{2} r_{C, D}(r)+2 q s_{C, D}(r)^{2}\right] .
\end{aligned}
$$


Proof. Since $\chi$ has conductor $q$, we have $\chi\left(C^{2}-\left(n^{2}-2\right) D^{2}\right)=\chi\left(C^{2}-\right.$ $\left.\left(r^{2}-2\right) D^{2}\right)$ for $n=q k+r$. We note that $s_{C, D}(q k+r)=s_{C, D}(r), r_{C, D}(q k+r)$ $=r_{C, D}(r)$ and $w_{C, D}(q k+r)=s_{C, D}(r) k+w_{C, D}(r)$. This proves the corollary.

3. Computation of special values of the zeta function associated with $\mathbb{Q}\left(\sqrt{n^{2}+2}\right)$. Let $d=n^{2}+2$ be a positive square free integer, and $K=\mathbb{Q}(\sqrt{d})$. Then the fundamental unit $\epsilon$ of $K$ is $n^{2}+1+n \sqrt{n^{2}+2}$ (see [8]). Let $\alpha=n+\sqrt{n^{2}+2}$. We note that $N_{K}(\epsilon)=1$ and $N_{K}(\alpha)=-2<0$. So if $h(d)=1$ then

$$
I(K)=(q) \cdot i\left(K^{+}\right) \cup(q \alpha) \cdot i\left(K^{+}\right),
$$

where $I(K)$ and $i\left(K^{+}\right)$are defined in the previous section. So we have

$$
\zeta_{K}(s, \chi)=\sum_{\substack{\mathbf{a} \in(q) \cdot i\left(K^{+}\right) \\ \text {integral }}} \frac{\chi(N(\mathbf{a}))}{N(\mathbf{a})^{s}}+\sum_{\substack{\mathbf{a} \in(q \alpha) \cdot i\left(K^{+}\right) \\ \text {integral }}} \frac{\chi(N(\mathbf{a}))}{N(\mathbf{a})^{s}} .
$$

We define

$R(\mathbf{b}):=\{a+b \epsilon \mid a, b \in \mathbb{Q}$ with $0<a \leq 1,0 \leq b<1$ and $\mathbf{b} \cdot(a+b \epsilon) \subset O(K)\}$ for an integral ideal $\mathbf{b}$ in $K$. In the following lemma, we find the set of all $(x, y)$ such that $x+y \epsilon \in R((q))$ or $x+y \epsilon \in R((q \alpha))$.

LEMMA 3.1.

$$
\begin{aligned}
\{(x, y) \mid x+y \epsilon & \in R((q))\}=\left\{(x, y) \mid x=\frac{C}{q}-\frac{D+q j}{q n}+\delta_{1}(j),\right. \\
y & \left.=\frac{D+q j}{q n} \text { for } j=0,1, \ldots, n-1 \text { and } 0 \leq C, D \leq q-1\right\},
\end{aligned}
$$

where

$$
\delta_{1}(j)= \begin{cases}1 & \text { if }\left\lceil\frac{C n-D}{q}\right\rceil \leq j \leq n-1, \\ 0 & \text { if } 0 \leq j \leq\left\lceil\frac{C n-D}{q}\right\rceil-1 .\end{cases}
$$

(ii) $\{(x, y) \mid x+y \epsilon \in R((q \alpha))\}=\left\{(x, y) \mid x=-\frac{C+q i}{2 n q}+\frac{t_{C, D}(n)}{q}+\delta_{2}(i)\right.$,

$$
\left.y=\frac{C+q i}{2 q n} \text { for } i=0,1, \ldots, 2 n-1 \text { and } 0 \leq C, D \leq q-1\right\},
$$

where

$$
\delta_{2}(i)= \begin{cases}0 & \text { if } 0 \leq i \leq\left\lceil\frac{2 n t_{C, D}(n)-C}{q}\right\rceil-1, \\ 1 & \text { if }\left\lceil\frac{2 n t_{C, D}(n)-C}{q}\right\rceil \leq i \leq 2 n-1,\end{cases}
$$

and $t_{C, D}(n)=D-C n-q\left[\frac{D-C n}{q}\right]$. 
Proof. The set $\{C+D \alpha \mid 0 \leq C, D \leq q-1\}$ is a complete set of representatives of $O_{K} / q O_{K}$, since $\{1, \alpha\}$ is a basis for $O_{K}$. So for $x+y \epsilon$ in $R((q))$ we have

$$
q(x+y \epsilon)=(C+D \alpha)+q(i+j \alpha)
$$

and for $x+y \epsilon$ in $R((q \alpha))$ we also have

$$
q \alpha(x+y \epsilon)=(C+D \alpha)+q(i+j \alpha),
$$

for some $0 \leq C, D \leq q-1$ and integers $i, j$. From the equation $\bar{\alpha}=\frac{2 n^{2}+1-\epsilon}{n}$ and computations similar to those in Lemmas 2.3 and 2.4, we obtain the assertion.

Proposition 3.2. If $h\left(n^{2}+2\right)=1$, then

$$
\begin{aligned}
\zeta_{K}(0, \chi) & \sum_{0 \leq C, D \leq q-1} \chi\left(C^{2}-2 D^{2}+2 n C D\right)\left[\sum_{j=0}^{n-1} S\left(\frac{C}{q}-\frac{D+q j}{q n}+\delta_{1}(j), \frac{D+q j}{q n}\right)\right. \\
& \left.-\sum_{i=0}^{2 n-1} S\left(\frac{t_{C, D}(n)}{q}-\frac{C+i q}{2 q n}+\delta_{2}(i), \frac{C+i q}{2 q n}\right)\right],
\end{aligned}
$$

where $\delta_{1}, \delta_{2}$ and $t_{C, D}$ are defined in Lemma 3.1, and $S(x, y)$ in Proposition 2.5.

Proof. As in Proposition 2.5, we have

$$
\begin{aligned}
\zeta_{K}(0, \chi)= & \sum_{x+y \epsilon \in R((q))} \chi\left(N_{K}(q(x+y \epsilon))\right) S(x, y) \\
& -\sum_{x+y \in \in R((\alpha q))} \chi\left(N_{K}(q \alpha(x+y \epsilon))\right) S(x, y) .
\end{aligned}
$$

For $x+y \epsilon \in R((q))$,

$$
q(x+y \epsilon)=C+D \alpha+q(i+j \alpha) .
$$

Thus

$$
N_{K}(q(x+y \epsilon)) \equiv N_{K}(C+D \alpha)(\bmod q)=C^{2}-2 D^{2}+2 n C D .
$$

If $x+y \epsilon$ is in $R((q \alpha))$, then

$$
q \alpha(x+y \epsilon)=C+D \alpha+q(i+j \alpha)
$$

for some $0 \leq C, D \leq q-1$ and integers $i, j$. So

$$
N_{K}(q \alpha(x+y \epsilon)) \equiv N_{K}(C+D \alpha)(\bmod q)=C^{2}-2 D^{2}+2 n C D .
$$

From (29)-(31), we obtain the conclusion immediately.

Finally, we obtain the following theorem. 
Theorem 3.3. If $h\left(n^{2}+2\right)=1$, then $\zeta_{K}(0, \chi)$

$$
\begin{aligned}
= & \frac{1}{12 q^{2}} \sum_{0 \leq C, D \leq q-1} \chi\left(C^{2}-2 D^{2}+2 n C D\right)\left(6 q^{2} A_{C, D}(n)+2 q^{2} n+3 C^{2} n\right. \\
& -12 q A_{C, D}(n) C+6 D^{2} n-6 q D n-6 n t_{C, D}(n)^{2}-6 q n t_{C, D}(n) \\
& \left.-3 n e_{C, D}(n)^{2}+3 q n e_{C, D}(n)-6 q^{2} B_{C, D}(n)+12 q B_{C, D}(n) t_{C, D}(n)+3 q C n\right),
\end{aligned}
$$

where $A_{C, D}(n)=\left\lceil\frac{n C-D}{q}\right\rceil, B_{C, D}(n)=\left\lceil\frac{2 n t_{C, D}(n)-C}{q}\right\rceil$ and $e_{C, D}(n)=C-$ $2 n t_{C, D}(n)+q B_{C, D}(n)$.

Proof. The equation $D-n C+q A_{C, D}(n)=t_{C, D}(n)$ implies that

$$
\begin{aligned}
\frac{A_{C, D}(n)^{2} n}{2}+\frac{D^{2} n}{2 q^{2}}-\frac{C D n^{2}}{q^{2}} & +\frac{C^{2} n^{3}}{2 q^{2}}+\frac{A_{C, D}(n) D n}{q}-\frac{A_{C, D}(n) C n^{2}}{q} \\
= & \frac{n\left(D-n C+q A_{C, D}(n)\right)^{2}}{2 q^{2}}=\frac{n t_{C, D}(n)^{2}}{2 q^{2}}
\end{aligned}
$$

and

$$
\frac{A_{C, D}(n) n}{2}+\frac{D n}{2 q}-\frac{C n^{2}}{2 q}=\frac{n\left(D-n C+q A_{C, D}(n)\right)}{2 q}=\frac{n t_{C, D}(n)}{2 q} .
$$

By (32) and (33), we have

$$
\sum_{j=0}^{n-1} S\left(\frac{C}{q}-\frac{D+q j}{n q}+\delta_{1}(j), \frac{D+q j}{q n}\right)
$$

$=\left(\frac{A_{C, D}(n)^{2} n}{2}+\frac{D^{2} n}{2 q^{2}}-\frac{C D n^{2}}{q^{2}}+\frac{C^{2} n^{3}}{2 q^{2}}+\frac{A_{C, D}(n) D n}{q}-\frac{A_{C, D}(n) C n^{2}}{q}\right)$

$-\left(\frac{A_{C, D}(n) n}{2}+\frac{D n}{2 q}-\frac{C n^{2}}{2 q}\right)+\frac{A_{C, D}(n)}{2}+\frac{n}{12}+\frac{C^{2} n}{2 q^{2}}-\frac{A_{C, D}(n) C}{q}$

$$
+\frac{D^{2} n}{2 q^{2}}-\frac{D n}{2 q}
$$

$$
=\frac{n t_{C, D}(n)^{2}}{2 q^{2}}-\frac{n t_{C, D}(n)}{2 q}+\frac{A_{C, D}(n)}{2}+\frac{n}{12}+\frac{C^{2} n}{2 q^{2}}-\frac{A_{C, D}(n) C}{q}+\frac{D^{2} n}{2 q^{2}}-\frac{D n}{2 q} .
$$

Also $C-2 n t_{C, D}(n)+q B_{C, D}(n)=e_{C, D}(n)$ implies that

$$
\begin{array}{r}
\frac{B_{C, D}(n)^{2} n}{4}+\frac{C^{2} n}{4 q^{2}}+\frac{B_{C, D}(n) C n}{2 q}-\frac{C n^{2} t_{C, D}(n)}{q^{2}}-\frac{B_{C, D}(n) n^{2} t_{C, D}(n)}{q} \\
+\frac{n^{3} t_{C, D}(n)^{2}}{q^{2}}=\frac{n\left(C-2 n t_{C, D}(n)+q B_{C, D}(n)\right)^{2}}{4 q^{2}}=\frac{n e_{C, D}(n)^{2}}{4 q^{2}},
\end{array}
$$


$(36)$

$$
\begin{aligned}
\frac{B_{C, D}(n) n}{4}+\frac{C n}{4 q}-\frac{n^{2} t_{C, D}(n)}{2 q} & =\frac{n\left(C-2 n t_{C, D}(n)+q B_{C, D}(n)\right)}{4 q} \\
& =\frac{n e_{C, D}(n)}{4 q} .
\end{aligned}
$$

From (35) and (36), we deduce that

$$
\sum_{i=0}^{2 n-1} S\left(\frac{t_{C, D}(n)}{q}-\frac{C+q i}{2 n q}+\delta_{2}(i), \frac{C+i q}{2 n q}\right)
$$

$$
\begin{aligned}
= & \frac{B_{C, D}(n)}{2}-\frac{n}{12}-\frac{B_{C, D}(n) n}{4}+\frac{B_{C, D}(n)^{2} n}{4}+\frac{C^{2} n}{2 q^{2}}-\frac{C n}{2 q}+\frac{B_{C, D}(n) C n}{2 q} \\
& -\frac{C n^{2} t_{C, D}(n)}{q^{2}}-\frac{B_{C, D}(n) t_{C, D}(n)}{q}+\frac{n^{2} t_{C, D}(n)}{2 q}-\frac{B_{C, D}(n) n^{2} t_{C, D}(n)}{q} \\
& +\frac{n t_{C, D}(n)^{2}}{q^{2}}+\frac{n^{3} t_{C, D}(n)^{2}}{q^{2}} \\
= & \frac{B_{C, D}(n)^{2} n}{4}+\frac{C^{2} n}{4 q^{2}}+\frac{B_{C, D}(n) C n}{2 q}-\frac{C n^{2} t_{C, D}(n)}{q^{2}}-\frac{B_{C, D}(n) n^{2} t_{C, D}(n)}{q} \\
& \left.+\frac{n^{3} t_{C, D}(n)^{2}}{q^{2}}\right)-\left(\frac{B_{C, D}(n) n}{4}+\frac{C n}{4 q}-\frac{n^{2} t_{C, D}(n)}{2 q}\right)+\frac{B_{C, D}(n)}{2}-\frac{n}{12} \\
& -\frac{B_{C, D}(n) t_{C, D}(n)}{q}+\frac{n t_{C, D}(n)^{2}}{q^{2}}+\frac{C^{2} n}{4 q^{2}}-\frac{C n}{4 q} \\
= & \frac{n e_{C, D}(n)^{2}}{4 q^{2}}-\frac{n e_{C, D}(n)}{4 q}+\frac{B_{C, D}(n)}{2}-\frac{n}{12} \\
& -\frac{B_{C, D}(n) t_{C, D}(n)}{q}+\frac{n t_{C, D}(n)^{2}}{q^{2}}+\frac{C^{2} n}{4 q^{2}}-\frac{C n}{4 q} .
\end{aligned}
$$

By combining Proposition 3.2, (34) and (37), we obtain the assertion.

COROLlary 3.4. If $h\left(n^{2}+2\right)=1$ and $n=q k+r$ for $r=0,1, \ldots, q-1$, then

where

$$
\zeta_{K}(0, \chi)=\frac{1}{12 q^{2}}\left(F_{\chi}(r) k+E_{\chi}(r)\right) \text {, }
$$

$$
\begin{aligned}
& E_{\chi}(r)=\sum_{0 \leq C, D \leq q-1} \chi\left(C^{2}-2 D^{2}+2 r C D\right)\left(6 q^{2} A_{C, D}(r)+2 q^{2} r+3 C^{2} r\right. \\
& -12 q A_{C, D}(r) C+6 D^{2} r-6 q D r-6 r t_{C, D}(r)^{2}-6 q r t_{C, D}(r)-3 r e_{C, D}(r)^{2} \\
& \left.+3 q r e_{C, D}(r)-6 q^{2} B_{C, D}(r)+12 q B_{C, D}(r) t_{C, D}(r)+3 q C r\right), \\
& F_{\chi}(r)=\sum_{0 \leq C, D \leq q-1} \chi\left(C^{2}-2 D^{2}+2 r C D\right)\left(-9 C^{2} q+6 D^{2} q-3 e_{C, D}(r)^{2} q\right. \\
& \left.\quad+9 C q^{2}-6 D q^{2}+3 e_{C, D}(r) q^{2}+2 q^{3}-18 q^{2} t_{C, D}(r)+18 q t_{C, D}(r)^{2}\right)
\end{aligned}
$$

and $A_{C, D}, B_{C, D}, t_{C, D}$ and $e_{C, D}$ are as in Theorem 3.3. 
Proof. We note that $A_{C, D}(q k+r)=C k+A_{C, D}(r), B_{C, D}(q k+r)=$ $2 t_{C, D}(r) k+B_{C, D}(r), t_{C, D}(q k+r)=t_{C, D}(r)$ and $e_{C, D}(q k+r)=e_{C, D}(r)$. Since the character $\chi$ has conductor $q$, the above equations yield the conclusion.

4. Proof of Theorem 1.1. Let $d=n^{2} \pm 2$ be a positive square free integer and $K=\mathbb{Q}(\sqrt{d})$. Let $q$ be a positive integer with $(q, d)=1, \chi$ an odd primitive character with conductor $q$ and $L_{\chi}$ a field over $\mathbb{Q}$ generated by the values of $\chi(a)$ for $a=1, \ldots, q$. Define $m_{\chi}:=\sum_{a=1}^{q} a \chi(a)$. Then from the same argument in Section 2 of [1] and Corollaries 2.9 and 3.4, we infer that if $n=q k+r$ and $h\left(n^{2}-2\right)=1$ with $n>q$, then

$$
B_{\chi}(r) k+A_{\chi}(r) \equiv 0(\bmod I),
$$

and if $h\left(n^{2}+2\right)=1$, then

$$
F_{\chi}(r) k+E_{\chi}(r) \equiv 0(\bmod I),
$$

for a prime ideal $I$ of $L_{\chi}$ dividing the principal ideal $\left(m_{\chi}\right)$. If the integers $q$ and $p$ satisfy condition $(*)$ in [6], then for $r$ such that $B_{\chi}(r) \notin I$ [resp. $\left.F_{\chi}(r) \notin I\right]$, there exists a unique $T_{A, B}^{\chi}(r)\left[\right.$ resp. $\left.T_{E, F}^{\chi}(r)\right] \in\{0,1, \ldots, p-1\}$ such that

$$
-q \frac{A_{\chi}(r)}{B_{\chi}(r)}+r+I=T_{A, B}^{\chi}(r)+I, \quad-q \frac{E_{\chi}(r)}{F_{\chi}(r)}+r+I=T_{E, F}^{\chi}(r)+I .
$$

So we have

(38) $n \equiv T_{A, B}^{\chi}(r)(\bmod p)$ for $n=q k+r$ with $h\left(n^{2}-2\right)=1$ and $n>q$,

(39) $n \equiv T_{E, F}^{\chi}(r)(\bmod p)$ for $n=q k+r$ with $h\left(n^{2}+2\right)=1$.

We will write $q \rightarrow p$ if $q$ and $p$ satisfy condition $(*)$ in [6]. From Section 4 in [1], we have

$$
175 \rightarrow 61, \quad 61 \rightarrow 1861, \quad 175 \rightarrow 1861 .
$$

Now, we find other $p$ and $q$ satisfying condition $(*)$. Consider the function $f_{25}:(\mathbb{Z} / 25 \mathbb{Z})^{*} \rightarrow \mathbb{Z} / 20 \mathbb{Z}$ for which $2^{f_{25}(a)} \equiv a(\bmod 25)$ and the function $g_{7}:(\mathbb{Z} / 7 \mathbb{Z})^{*} \rightarrow \mathbb{Z} / 6 \mathbb{Z}$ for which $3^{g 7(a)} \equiv a(\bmod 7)$. These two functions are well defined, since $(\mathbb{Z} / 25 \mathbb{Z})^{*}\left[\right.$ resp. $\left.(\mathbb{Z} / 7 \mathbb{Z})^{*}\right]$ is a cyclic group generated by 2 [resp. 3]. Define

$$
\chi_{4}:(\mathbb{Z} / 175 \mathbb{Z})^{*} \rightarrow \mathbb{C}
$$

by $\chi_{4}(a)=\zeta_{30}^{6 f_{25}\left(a_{25}\right)} \cdot \zeta_{30}^{25 g_{7}\left(a_{7}\right)}$, where $a \equiv a_{25}(\bmod 25), a \equiv a_{7}(\bmod 7)$ and $\zeta_{30}$ is a primitive 30 th root of unity. Then $\chi_{4}$ is an odd primitive character with conductor 175 . Since the order of 450 modulo 601 is $30, I_{4}=(601$, $\left.\zeta_{30}-450\right)$ is the prime ideal in $L_{\chi_{4}}=\mathbb{Q}\left(\zeta_{30}\right)$ lying over the rational prime 601 of degree 1 (see page 97 in [1]). From $\zeta_{30} \equiv 450\left(\bmod I_{4}\right)$, we find that

$$
m_{\chi_{4}} \equiv 0\left(\bmod I_{4}\right) \text {. }
$$


So we obtain

$$
175 \rightarrow 601 \text {. }
$$

Now we define the functions $T_{A, B}^{\chi_{i}}(r)$ as follows:

$$
\begin{array}{r}
-175 \frac{A_{\chi_{1}}(r)}{B_{\chi_{1}}(r)}+r+I_{1}=T_{A, B}^{\chi_{1}}(r)+I_{1}, \\
-61 \frac{A_{\chi_{2}}(r)}{B_{\chi_{2}}(r)}+r+I_{2}=T_{A, B}^{\chi_{2}}(r)+I_{2}, \\
-175 \frac{A_{\chi_{3}}(r)}{B_{\chi_{3}}(r)}+r+I_{3}=T_{A, B}^{\chi_{3}}(r)+I_{3}, \\
-175 \frac{A_{\chi_{4}}(r)}{B_{\chi_{4}}(r)}+r+I_{4}=T_{A, B}^{\chi_{4}}(r)+I_{4},
\end{array}
$$

where the characters $\chi_{i}$ and ideals $I_{i}$ are defined in Examples 1, 3 and 2 of Section 4 in [1], respectively, for $i=1,2,3$.

For a residue $a_{175}$ modulo 175 with $B_{\chi_{1}}\left(a_{175}\right) \notin I_{1}\left[\operatorname{resp} . B_{\chi_{3}}\left(a_{175}\right) \notin I_{3}\right]$, we define $b_{61}$ [resp. $d_{1861}$ ] to be residues modulo 61 [resp. 1861] for which

$$
b_{61}=T_{A, B}^{\chi_{1}}\left(a_{175}\right), \quad d_{1861}=T_{A, B}^{\chi_{3}}\left(a_{175}\right) .
$$

And for a residue $b_{61}$ modulo 61 with $B_{\chi_{2}}\left(b_{61}\right) \notin I_{2}$, we define $c_{1861}$ to be a residue modulo 1861 such that

$$
c_{1861}=T_{A, B}^{\chi_{2}}\left(b_{61}\right) .
$$

Let $U_{m}=\left\{a \in \mathbb{Z} \mid\left(\frac{a^{2}-2}{p}\right)=-1\right.$ for any prime $p$ dividing $\left.m\right\}$. By computer we can also check that if $a_{175} \in U_{175}$ then $B_{\chi_{1}}\left(a_{175}\right) \notin I_{1}$ and $B_{\chi_{3}}\left(a_{175}\right) \notin I_{3}$, and if $b_{61}=T_{A, B}^{\chi_{1}}\left(a_{175}\right)$ for $a_{175} \in U_{175}$ then $B_{\chi_{2}}\left(b_{61}\right) \notin I_{2}$. Hence it is possible to calculate $T_{A, B}^{\chi_{1}}\left(a_{175}\right), T_{A, B}^{\chi_{3}}\left(a_{175}\right)$ and $T_{A, B}^{\chi_{2}}\left(b_{61}\right)$ for $a_{175} \in U_{175}$. From the computer calculations, we obtain the following table:

\begin{tabular}{cccccccc}
\hline$a_{175} \in U_{175}$ & $b_{61}$ & $c_{1861}$ & $d_{1861}$ & $a_{175} \in U_{175}$ & $b_{61}$ & $c_{1861}$ & $d_{1861}$ \\
\hline \pm 7 & \pm 7 & \pm 7 & \pm 7 & \pm 8 & \pm 8 & \pm 8 & \pm 8 \\
\pm 13 & \pm 13 & \pm 13 & \pm 13 & \pm 15 & \pm 38 & \pm 1266 & \pm 1060 \\
\pm 20 & \pm 20 & \pm 20 & \pm 20 & \pm 22 & \pm 23 & \pm 595 & \pm 1022 \\
\pm 27 & \pm 34 & \pm 851 & \pm 389 & \pm 28 & \pm 59 & \pm 1859 & \pm 962 \\
\pm 35 & \pm 51 & \pm 1851 & \pm 288 & \pm 42 & \pm 43 & \pm 329 & \pm 392 \\
\pm 43 & \pm 40 & \pm 1821 & \pm 1353 & \pm 48 & \pm 34 & \pm 851 & \pm 306 \\
\pm 50 & \pm 16 & \pm 1075 & \pm 193 & \pm 55 & \pm 35 & \pm 1272 & \pm 566 \\
\pm 57 & \pm 32 & \pm 845 & \pm 1559 & \pm 62 & \pm 6 & \pm 301 & \pm 1647 \\
\pm 63 & \pm 43 & \pm 329 & \pm 399 & \pm 70 & \pm 58 & \pm 1858 & \pm 49 \\
\pm 77 & \pm 4 & \pm 4 & \pm 1760 & \pm 78 & \pm 9 & \pm 1690 & \pm 561 \\
\pm 83 & \pm 26 & \pm 589 & \pm 427 & \pm 85 & \pm 49 & \pm 1501 & \pm 1072 \\
\hline
\end{tabular}


Also for $r$ with $F_{\chi_{i}}(r) \notin I_{i}$, the functions $T_{E, F}^{\chi_{i}}(r)$ are defined as follows:

$$
\begin{array}{r}
-175 \frac{E_{\chi_{1}}(r)}{F_{\chi_{1}}(r)}+r+I_{1}=T_{E, F}^{\chi_{1}}(r)+I_{1}, \\
-61 \frac{E_{\chi_{2}}(r)}{F_{\chi_{2}}(r)}+r+I_{2}=T_{E, F}^{\chi_{2}}(r)+I_{2}, \\
-175 \frac{E_{\chi_{3}}(r)}{F_{\chi_{3}}(r)}+r+I_{3}=T_{E, F}^{\chi_{3}}(r)+I_{3}, \\
-175 \frac{E_{\chi_{4}}(r)}{F_{\chi_{4}}(r)}+r+I_{4}=T_{E, F}^{\chi_{4}}(r)+I_{4} .
\end{array}
$$

Then for a residue $e_{175}$ modulo 175 with $F_{\chi_{1}}\left(e_{175}\right) \notin I_{1}$ [resp. $F_{\chi_{3}}\left(e_{175}\right)$ $\notin I_{3}$ ], we define $f_{61}$ [resp. $h_{1861}$ ] to be residues modulo 61 [resp. 1861] for which

$$
f_{61}=T_{E, F}^{\chi_{1}}\left(e_{175}\right), \quad h_{1861}=T_{E, F}^{\chi_{3}}\left(e_{175}\right) .
$$

And for a residue $f_{61}$ modulo 61 with $F_{\chi_{2}}\left(f_{61}\right) \notin I_{2}$, we define $g_{1861}$ to be a residue modulo 1861 such that

$$
g_{1861}=T_{E, F}^{\chi_{2}}\left(f_{61}\right) .
$$

Let $V_{m}=\left\{a \in \mathbb{Z} \mid\left(\frac{a^{2}+2}{p}\right)=-1\right.$ for any prime $p$ dividing $\left.m\right\}$. Then by computer we can also check that if $e_{175} \in V_{175}$ then $F_{\chi_{1}}\left(e_{175}\right) \notin I_{1}$ and $F_{\chi_{3}}\left(e_{175}\right) \notin I_{3}$, and if $f_{61}=T_{E, F}^{\chi_{1}}\left(e_{175}\right)$ for $e_{175} \in V_{175}$ then $F_{\chi_{2}}\left(f_{61}\right) \notin I_{2}$. So we can calculate $T_{E, F}^{\chi_{1}}\left(e_{175}\right), T_{E, F}^{\chi_{3}}\left(e_{175}\right)$ and $T_{E, F}^{\chi_{2}}\left(f_{61}\right)$ for $e_{175} \in V_{175}$. We obtain the following table:

\begin{tabular}{cccccccc}
\hline$e_{175} \in V_{175}$ & $f_{61}$ & $g_{1861}$ & $h_{1861}$ & $e_{175} \in V_{175}$ & $f_{61}$ & $g_{1861}$ & $h_{1861}$ \\
\hline \pm 1 & \pm 1 & \pm 1 & \pm 1 & \pm 5 & \pm 5 & \pm 5 & \pm 5 \\
\pm 6 & \pm 6 & \pm 6 & \pm 6 & \pm 9 & \pm 9 & \pm 9 & \pm 9 \\
\pm 15 & \pm 15 & \pm 15 & \pm 15 & \pm 16 & \pm 50 & \pm 491 & \pm 935 \\
\pm 19 & \pm 19 & \pm 244 & \pm 1534 & \pm 20 & \pm 44 & \pm 403 & \pm 943 \\
\pm 26 & \pm 41 & \pm 1235 & \pm 1243 & \pm 29 & \pm 19 & \pm 244 & \pm 1567 \\
\pm 30 & \pm 24 & \pm 610 & \pm 363 & \pm 34 & \pm 12 & \pm 32 & \pm 1589 \\
\pm 36 & \pm 28 & \pm 458 & \pm 200 & \pm 40 & \pm 30 & \pm 1654 & \pm 578 \\
\pm 41 & \pm 21 & \pm 804 & \pm 1762 & \pm 44 & \pm 30 & \pm 1654 & \pm 186 \\
\pm 50 & \pm 47 & \pm 1124 & \pm 213 & \pm 51 & \pm 45 & \pm 728 & \pm 181 \\
\pm 54 & \pm 54 & \pm 778 & \pm 1097 & \pm 55 & \pm 39 & \pm 240 & \pm 40 \\
\pm 61 & \pm 51 & \pm 753 & \pm 858 & \pm 64 & \pm 25 & \pm 155 & \pm 817 \\
\pm 65 & \pm 4 & \pm 4 & \pm 1691 & \pm 69 & \pm 26 & \pm 1280 & \pm 784 \\
\pm 71 & \pm 27 & \pm 190 & \pm 339 & \pm 75 & \pm 60 & \pm 1860 & \pm 70 \\
\pm 76 & \pm 57 & \pm 1857 & \pm 651 & \pm 79 & \pm 42 & \pm 1617 & \pm 1056 \\
\pm 85 & \pm 44 & \pm 403 & \pm 1623 & \pm 86 & \pm 8 & \pm 1448 & \pm 1048 \\
\hline
\end{tabular}


To prove our theorem, we need the following class number 1 criteria.

LEMMA 4.1.

(i) $h\left((2 k+1)^{2}-2\right)=1 \Rightarrow 4 k^{2}+4 k-1-4 t^{2}$ are primes for $0 \leq t \leq k$,

(ii) $h\left(4 k^{2}-2\right)=1 \Rightarrow 2 k^{2}-1-2 t^{2}$ are primes for $0 \leq t \leq k-1$,

(iii) $h\left((2 k+1)^{2}+2\right)=1 \Rightarrow(2 k+1)^{2}+2-4 t^{2}$ are primes for $0 \leq t \leq k$,

(iv) $h\left(4 k^{2}+2\right)=1 \Rightarrow 4 k^{2}+2-(2 t-1)^{2}$ are primes for $1 \leq t \leq k$.

Proof. See Corollaries 3.3-3.6 in [4]. (Remark: $d=(2 n+1)^{2}-2$ in Corollary 3.5 of [4] is a misprint. It should read $d=(2 n+1)^{2}+2$.)

In the following proposition, we find an upper bound for $n$ with $h\left(n^{2}-2\right)$ $=1$.

Proposition 4.2. Let $n^{2}-2$ be a square free integer. Then $h\left(n^{2}-2\right)$ $>1$ for $n>1244$.

Proof. If $n \notin U_{175}$ and $n=2 i+1$, then there exists an integer $t_{0} \in \mathbb{Z}$ such that $(2 i+1)^{2}-2-\left(2 t_{0}\right)^{2} \equiv 0(\bmod 5)$ or $(2 i+1)^{2}-2-\left(2 t_{0}\right)^{2} \equiv 0$ $(\bmod 7)$. Similarly, for $n=2 j \notin U_{175}$, there is an integer $s_{0} \in \mathbb{Z}$ such that $2 j^{2}-1-2 s_{0}^{2} \equiv 0(\bmod 5)$ or $2 j^{2}-1-2 s_{0}^{2} \equiv 0(\bmod 7)$. If we take $t_{0}=4$ for $i=5 l, 5 l+4, t_{0}=5$ for $i=7 l+4,7 l+2$ and $t_{0}=7$ for $i=7 l+1,7 l+5$, then $(2 i+1)^{2}-2-\left(2 t_{0}\right)^{2}$ is a multiple of 5 or 7 . And if we take $s_{0}=4$ for $j=5 l+3,5 l+2, s_{0}=5$ for $j=7 l+1,7 l+6$ and $s_{0}=7$ for $j=7 l+5,7 l+2$ then $2 j^{2}-1-2 s_{0}^{2}$ is a multiple of 5 or 7 . Thus from Lemma 4.1(i), (ii), we have

$$
h\left(n^{2}-2\right)>1 \quad \text { for } n>15 \text { with } n \notin U_{175} .
$$

Suppose $n \equiv a_{175}(\bmod 175)$ for $a_{175} \in U_{175}$ and $a_{175} \neq \pm 7, \pm 8, \pm 13, \pm 20$ and $h\left(n^{2}-2\right)=1, n>175$. Then from the table we have $c_{1861} \neq d_{1861}$. This is a contradiction to (38). So

$$
\begin{aligned}
& h\left(n^{2}-2\right)>1 \quad \text { for } n \not \equiv \pm 7, \pm 8, \pm 13, \pm 20(\bmod 175) \text { and } \\
& \quad n \in U_{175} \text { with } n>175 .
\end{aligned}
$$

By computer, we find $T_{A, B}^{\chi_{4}}( \pm 20)= \pm 20$. That is, $n \equiv \pm 20(\bmod 601)$ for $n \equiv \pm 20(\bmod 175)$ with $h\left(n^{2}-2\right)=1$ and $n>175$. If we take $t_{0}=20$, then $(2 i+1)^{2}-2-\left(2 t_{0}\right)^{2}$ is a multiple of 601 for $2 i+1 \equiv \pm 20(\bmod 601)$. And if we also take $s_{0}=621$ then $2 j^{2}-1-2 s_{0}^{2}$ is a multiple of 601 for $2 j \equiv \pm 20(\bmod 601)$. Thus by Lemma 4.1(i), (ii), we have

$$
h\left(n^{2}-2\right)>1 \quad \text { for } n \equiv \pm 20(\bmod 175) \text { with } n>1244 \text {. }
$$

If $n \equiv \pm 7, \pm 8$ or $\pm 13(\bmod 175)$ then $n \in U_{175}$ but if we assume $n>175$ and $h\left(n^{2}-2\right)=1$, then $n \equiv \pm 7, \pm 8$ or $\pm 13(\bmod 61)$. So $n \notin U_{61}$. We can find $t_{0}$ [resp. $s_{0}$ ] making $(2 i+1)^{2}-2-\left(2 t_{0}\right)^{2}$ [resp. $2 j^{2}-1-2 s_{0}^{2}$ ] a multiple of 61 for $2 i+1 \equiv \pm 7, \pm 8$ or $\pm 13(\bmod 61)[$ resp. $2 j \equiv \pm 7$, \pm 8 or $\pm 13(\bmod 61)]$ as above. They give an upper bound for $n$ such that 
$h\left(n^{2}-2\right)>1$, for $n \equiv \pm 7, \pm 8$ or $\pm 13(\bmod 175)$. The upper bound does not exceed 1244. So we have

$$
h\left(n^{2}-2\right)>1 \quad \text { for } n \equiv \pm 7, \pm 8, \pm 13(\bmod 175) \text { with } n>1244 .
$$

By (41)-(44), the proof is complete.

Now, we find an upper bound for $n$ with $h\left(n^{2}+2\right)=1$.

Proposition 4.3. Let $n^{2}+2$ be a square free integer. Then $h\left(n^{2}+2\right)>1$ for $n>1596$.

Proof. For $n \notin V_{175}$, it is possible to find $t_{0}$ [resp. $s_{0}$ ] such that $(2 i+1)^{2}$ $+2-\left(2 t_{0}\right)^{2}\left[\right.$ resp. $\left.(2 j)^{2}+2-\left(2 s_{0}-1\right)^{2}\right]$ is a multiple of 5 or 7 with $n=2 i+1$ [resp. $n=2 j$ ]. If we take $t_{0}=2$ for $i=5 l+3, i=7 l+3, t_{0}=7$ for $i=5 l+1$ and $t_{0}=6$ for $i=7 l+1,7 l+5$, then $(2 i+1)^{2}+2-\left(2 t_{0}\right)^{2}$ is a multiple of 5 or 7 . And if we take $s_{0}=6$ for $j=5 l+1,5 l+4,7 l$ and $s_{0}=5$ for $j=7 l+5,7 l+2$ then $2 j^{2}-1-2 s_{0}^{2}$ is a multiple of 5 or 7 . Thus from Lemma 4.1(iii), (v), we have

$$
h\left(n^{2}+2\right)>1 \quad \text { for } n>15 \text { and } n \notin V_{175} .
$$

If $n \equiv e_{175}(\bmod 175)$ for $e_{175} \in V_{175}$ and $e_{175} \neq \pm 1, \pm 5, \pm 6, \pm 9, \pm 15$ and $h\left(n^{2}+2\right)=1$, then from the table we have $g_{1861} \neq h_{1861}$. This is a contradiction to (39). So

$$
h\left(n^{2}+2\right)>1 \text { for } n \not \equiv \pm 1, \pm 5, \pm 6, \pm 9, \pm 15(\bmod 175) \text { and } n \in V_{175} .
$$

If $n \equiv \pm 6(\bmod 175)$ with $h\left(n^{2}+2\right)=1$, then $n \equiv \pm 6(\bmod 1861)$. Suppose $n \equiv \pm 6(\bmod 1861)$ and $n=2 i+1$. Then $i=1861 l+933$ or $1861 l+$ 927 . Take $t_{0}=133$. Then $(2 i+1)^{2}+2-\left(2 t_{0}\right)^{2}$ is $1861\left(7444 l^{2}+7468 l+1835\right)$ or $1861\left(7444 l^{2}+7420 l+1811\right)$. Suppose $n \equiv \pm 6(\bmod 1861)$ and $n=2 j$. Then $j=1861 l+3$ or $1861 l+1858$. If we take $s_{0}=798$ then $(2 j)^{2}+2-\left(2 s_{0}-1\right)^{2}=$ $1861\left(7444 l^{2}+24 l-1367\right)$ or $1861\left(7444 l^{2}+14864 l+6053\right)$. Thus by Lemma 4.1(iii), (v), we have

$$
h\left(n^{2}+2\right)>1 \quad \text { for } n \equiv \pm 6(\bmod 175) \text { with } n>1596 .
$$

If $n \equiv \pm 1, \pm 5, \pm 9(\bmod 175)$ then $n \in V_{175}$ but by assumption of $h\left(n^{2}+2\right)=1$, we have $n= \pm 1, \pm 5, \pm 9(\bmod 61)$. Moreover, $n \notin V_{61}$. So we can find $t_{0}$ [resp. $s_{0}$ ] such that $(2 i+1)^{2}+2-\left(2 t_{0}\right)^{2}$ [resp. $(2 j)^{2}+2-\left(2 s_{0}-1\right)^{2}$ ] are multiples of 61 for $2 i+1 \equiv \pm 1, \pm 5, \pm 9(\bmod 61)[$ resp. $2 j \equiv \pm 1, \pm 5, \pm 9$ $(\bmod 61)]$. They give an upper bound for $n$ such that $h\left(n^{2}+2\right)=1$ and $n \equiv \pm 1, \pm 5, \pm 9(\bmod 175)$ as above. From this, we have

$$
h\left(n^{2}+2\right)>1 \quad \text { for } n \equiv \pm 1, \pm 5, \pm 9(\bmod 175) \text { with } n>114 .
$$

By computer, we find $T_{A, B}^{\chi_{4}}( \pm 15)= \pm 15$. So if $n \equiv \pm 15(\bmod 175)$ with $h\left(n^{2}+2\right)=1$, then $n \equiv \pm 15(\bmod 601)$. If we take $t_{0}=105$ [resp. $s_{0}=196$ ] then $(2 i+1)^{2}+2-\left(2 t_{0}\right)^{2}$ [resp. $\left.(2 j)^{2}+2-\left(2 s_{0}-1\right)^{2}\right]$ is a multiple of 601 for 
any $2 i+1 \equiv \pm 15(\bmod 601)[$ resp. $2 j \equiv \pm 15(\bmod 601)]$. Thus by Lemma 4.1(iii), (v), we have

$$
h\left(n^{2}+2\right)>1 \quad \text { for } n \equiv \pm 15(\bmod 175) \text { with } n>392 .
$$

By (45)-(49), the proof is complete.

Proof of Mollin and Williams' Conjecture. By Propositions 4.2 and 4.3,

$$
h\left(n^{2} \pm 2\right)>1 \quad \text { for } n>1596 .
$$

In [12], Mollin and Williams prove that if $d=n^{2} \pm 2$ is a square free integer with $n<5000$ then

$$
h(d)=1 \quad \text { if and only if } \quad d=3,6,7,11,14,38,47,62,83,167,227,398 .
$$

By combining the above two results, we obtain the conjecture.

Now, we recall the following proposition.

Proposition 4.4 ([3]). Let d be a Richaud-Degert type.

I. Suppose $d=n^{2}+r \equiv 2,3(\bmod 4)$.

(i) If $|r| \neq 1,4$, then $h(d)>1$ except for $r= \pm 2$.

(ii) If $|r|=1$, then $h(d)>1$ except for $d=2,3$.

II. Suppose $d=n^{2}+r \equiv 1(\bmod 8)$.

(i) If $|r| \neq 1,4$, then $h(d)>1$ except for $d=33$.

(ii) If $|r|=1$, then $h(d)>1$ except for $d=17$.

Since Mollin and Williams' conjecture is true, Theorem 1.1 is a direct consequence of Proposition 4.4.

5. Appendix. In this section, we provide a MATHEMATICA program to evaluate the values $T_{A, B}^{\chi_{i}}(r)$ and $T_{E, F}^{\chi_{i}}(r)$ in Section 4.

The function $\mathrm{f}\left[\mathrm{x}_{-}, \mathrm{y}_{-}\right]$computes the logarithm of $x$ to base 2 modulo $y$, and $\mathrm{g}\left[\mathrm{x}_{-}, \mathrm{y}_{-}\right]$computes the logarithm of $x$ to base 3 modulo $y$ :

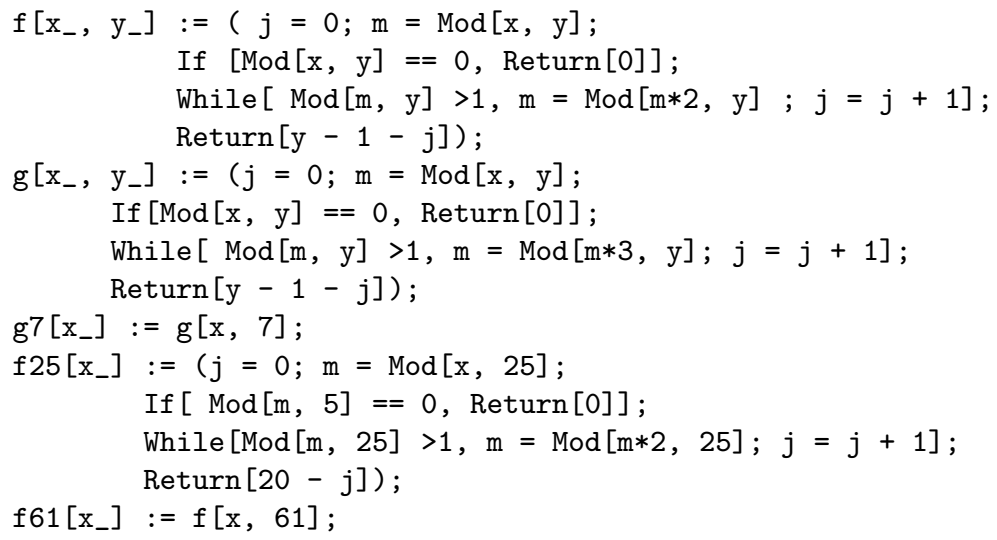


The function iv $\left[\mathrm{x}_{-}, \mathrm{y}\right]$ computes the multiplicative inverse of $x$ modulo $y$ :

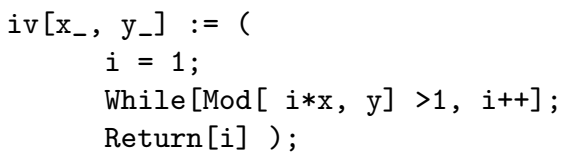

The functions chi [a_] compute $\chi_{i}(a)$ modulo $I_{i}$ for $i=1,2,3,4$ :

$\operatorname{ch} 1\left[\mathrm{a}_{-}\right]:=(\operatorname{If}[\operatorname{Mod}[\mathrm{a}, 5]==0|| \operatorname{Mod}[\mathrm{a}, 7]==0, \operatorname{Return}[0]]$;

Return [Mod [PowerMod [8, f25[Mod [a, 25] ] , 61]*

PowerMod [47, g7[Mod [a, 7] ] , 61] , 61] ]);

$\operatorname{ch} 2\left[a_{-}\right]:=(\operatorname{If}[\operatorname{Mod}[a, 61]==0$, Return [0] $]$;

Return [PowerMod [1833, f61[Mod [a, 61]] , 1861]]);

$\operatorname{ch} 3\left[a_{-}\right]:=(\operatorname{If}[\operatorname{Mod}[a, 5]==0|| \operatorname{Mod}[a, 7]==0$, Return[0]];

Return [Mod [PowerMod [380, f25[Mod [a, 25] ] , 1861] *

PowerMod [1406, g7[Mod[a, 7] ] , 1861], 1861]]);

$\operatorname{ch} 4\left[a_{-}\right]:=$

(If $[\operatorname{Mod}[a, 5]==0|| \operatorname{Mod}[a, 7]==0, \operatorname{Return}[0]]$;

Return [Mod [PowerMod [432, f25[Mod [a, 25] ], 601]*

PowerMod [577, g7 [Mod [a, 7] ] , 601], 601]]);

The following are needed to compute $A_{\chi_{i}}(r)$ and $B_{\chi_{i}}(r)$ modulo $I_{i}$ :

$\mathrm{u}\left[\mathrm{q}_{-}, \mathrm{n}_{-}, \mathrm{c}_{-}, \mathrm{d}_{-}\right]:=\mathrm{Floor}[(\mathrm{n} \mathrm{d}-\mathrm{c}) / \mathrm{q}]$

$r\left[q_{-}, n_{-}, c_{-}, d_{-}\right]:=n d-c-q F l o o r[(n d-c) / q]$

$\mathrm{v}\left[\mathrm{q}_{-}, \mathrm{n}_{-}, \mathrm{c}_{-}, \mathrm{d}_{-}\right]:=\mathrm{F} \operatorname{loor}[(\mathrm{r}[\mathrm{q}, \mathrm{n}, \mathrm{c}, \mathrm{d}] \mathrm{n}-\mathrm{d}) / \mathrm{q}]$

$\mathrm{s}\left[\mathrm{q}_{-}, \mathrm{n}_{-}, \mathrm{c}_{-}, \mathrm{d}_{-}\right]:=\mathrm{r}[\mathrm{q}, \mathrm{n}, \mathrm{c}, \mathrm{d}] \mathrm{n}-\mathrm{d}-\mathrm{q} \operatorname{Floor}[(\mathrm{r}[\mathrm{q}, \mathrm{n}, \mathrm{c}, \mathrm{d}] \mathrm{n}-\mathrm{d}) / \mathrm{q}]$

$\mathrm{w}\left[\mathrm{q}_{-}, \mathrm{n}_{-}, \mathrm{c}_{-}, \mathrm{d}_{-}\right]:=-\mathrm{F} \operatorname{loor}[(\mathrm{r}[\mathrm{q}, \mathrm{n}, \mathrm{c}, \mathrm{d}]-\mathrm{n} \mathrm{s}[\mathrm{q}, \mathrm{n}, \mathrm{c}, \mathrm{d}]) / \mathrm{q}]$

$A\left[q_{-}, n_{-}, c_{-}, d_{-}\right]:=-2 q^{\wedge} 2+\mathrm{n}^{\wedge} 2+3 \mathrm{qr}[\mathrm{q}, \mathrm{n}, \mathrm{c}, \mathrm{d}]-\mathrm{q} \mathrm{n}[\mathrm{q}, \mathrm{n}, \mathrm{c}, \mathrm{d}]$

$-q^{\wedge} 2 w[q, n, c, d]+3 q d+2 q w[q, n, c, d] s[q, n, c, d]$

$+2 \mathrm{n} r[\mathrm{q}, \mathrm{n}, \mathrm{c}, \mathrm{d}] \sim 2-2 \mathrm{~s}[\mathrm{q}, \mathrm{n}, \mathrm{c}, \mathrm{d}] \mathrm{r}[\mathrm{q}, \mathrm{n}, \mathrm{c}, \mathrm{d}]-4 \mathrm{~d} r[\mathrm{q}, \mathrm{n}, \mathrm{c}, \mathrm{d}]$

$-2 c d+2 n d^{\wedge} 2+q c-2 q n d+q s[q, n, c, d]-2 q n r[q, n, c, d]$

$B\left[q_{-}, n_{-}, c_{-}, d_{-}\right]:=q^{\wedge} 3-2 q^{\wedge} 2 s[q, n, c, d]+2 r[q, n, c, d] \wedge 2 q$

$+2 d^{\wedge} 2 q-2 q^{\wedge} 2 d-2 q^{\wedge} 2 r[q, n, c, d]+2 q s[q, n, c, d] \wedge 2$

$\mathrm{SB} 1\left[\mathrm{n}_{-}, \mathrm{c}_{-}, \mathrm{d}_{-}\right]:=\operatorname{Mod}\left[\operatorname{ch} 1\left[\mathrm{c}^{\wedge} 2-\left(\mathrm{n}^{\wedge} 2-2\right) \mathrm{d}^{\wedge} 2\right] * \mathrm{~B}[175, \mathrm{n}, \mathrm{c}, \mathrm{d}], 61\right]$

$\operatorname{SB} 2\left[n_{-}, c_{-}, d_{-}\right]:=\operatorname{Mod}\left[\operatorname{ch} 2\left[c^{\wedge} 2-\left(n^{\wedge} 2-2\right) d^{\wedge} 2\right] * B[61, n, c, d], 1861\right]$

$\mathrm{SB} 3\left[\mathrm{n}_{-}, \mathrm{c}_{-}, \mathrm{d}_{-}\right]:=\operatorname{Mod}\left[\mathrm{ch} 3\left[\mathrm{c}^{\wedge} 2-\left(\mathrm{n}^{\wedge} 2-2\right) \mathrm{d}^{\wedge} 2\right] * \mathrm{~B}[175, \mathrm{n}, \mathrm{c}, \mathrm{d}], 1861\right]$

SB4 $\left[\mathrm{n}_{-}, c_{-}, d_{-}\right]:=\operatorname{Mod}\left[\operatorname{ch} 4\left[c^{\wedge} 2-\left(\mathrm{n}^{\wedge} 2-2\right) d^{\wedge} 2\right] * B[175, \mathrm{n}, \mathrm{c}, \mathrm{d}], 601\right]$

$\operatorname{SA} 1\left[n_{-}, c_{-}, d_{-}\right]:=\operatorname{Mod}\left[\operatorname{ch} 1\left[c^{\wedge} 2-\left(n^{\wedge} 2-2\right) d^{\wedge} 2\right] * A[175, n, c, d], 61\right]$

$\mathrm{SA} 2\left[\mathrm{n}_{-}, c_{-}, \mathrm{d}_{-}\right]:=\operatorname{Mod}\left[\operatorname{ch} 2\left[\mathrm{c}^{\wedge} 2-\left(\mathrm{n}^{\wedge} 2-2\right) \mathrm{d}^{\wedge} 2\right] * \mathrm{~A}[61, \mathrm{n}, \mathrm{c}, \mathrm{d}], 1861\right]$

$\mathrm{SA} 3\left[\mathrm{n}_{-}, c_{-}, d_{-}\right]:=\operatorname{Mod}\left[\operatorname{ch} 3\left[c^{\wedge} 2-\left(\mathrm{n}^{\wedge} 2-2\right) \mathrm{d}^{\wedge} 2\right] * A[175, \mathrm{n}, \mathrm{c}, \mathrm{d}], 1861\right]$

$\mathrm{SA} 4\left[\mathrm{n}_{-}, \mathrm{c}_{-}, \mathrm{d}_{-}\right]:=\operatorname{Mod}\left[\operatorname{ch} 4\left[\mathrm{c}^{\wedge} 2-\left(\mathrm{n}^{\wedge} 2-2\right) \mathrm{d}^{\wedge} 2\right] * \mathrm{~A}[175, \mathrm{n}, \mathrm{c}, \mathrm{d}], 601\right]$

The functions RAi [a_] and RBi [a_] compute $A_{\chi_{i}}(a)$ and $B_{\chi_{i}}(a)$ modulo $I_{i}$ respectively, where $\mathrm{q}$ is the conductor for the character $\chi_{i}$ for $i=1,2,3,4$ :

RB1 [a_] : :

$\operatorname{Mod}[\operatorname{Sum}[\operatorname{Mod}[\operatorname{Sum}[\operatorname{SB1}[a, c, d],\{c, 0,174\}], 61],\{d, 0,174\}], 61]$ 
RA1 [a_] :=

$\operatorname{Mod}[\operatorname{Sum}[\operatorname{Mod}[\operatorname{Sum}[\operatorname{SA} 1[a, c, d],\{c, 0,174\}], 61],\{d, 0,174\}], 61]$

RB2 [a_] :=

$\operatorname{Mod}[\operatorname{Sum}[\operatorname{Mod}[\operatorname{Sum}[\operatorname{SB} 2[a, c, d],\{c, 0,60\}], 1861],\{d, 0,60\}], 1861]$

RA2 [a_] :=

$\operatorname{Mod}[\operatorname{Sum}[\operatorname{Mod}[\operatorname{Sum}[\operatorname{SA} 2[a, c, d],\{c, 0,60\}], 1861],\{d, 0,60\}], 1861]$

RB3 [a_] :=

$\operatorname{Mod}[\operatorname{Sum}[\operatorname{Mod}[\operatorname{Sum}[\mathrm{SB} 3[\mathrm{a}, \mathrm{c}, \mathrm{d}],\{\mathrm{c}, 0,174\}], 1861],\{\mathrm{d}, 0,174\}], 1861]$

RA3 [a_] : :

$\operatorname{Mod}[\operatorname{Sum}[\operatorname{Mod}[\operatorname{Sum}[\operatorname{SA} 3[a, c, d],\{c, 0,174\}], 1861],\{d, 0,174\}], 1861]$

$\mathrm{RB} 4\left[\mathrm{a}_{-}\right]:=$

$\operatorname{Mod}[\operatorname{Sum}[\operatorname{Mod}[\operatorname{Sum}[\operatorname{SB} 4[a, c, d],\{c, 0,174\}], 601],\{d, 0,174\}], 601]$

RA4 [a_] : =

$\operatorname{Mod}[\operatorname{Sum}[\operatorname{Mod}[\operatorname{Sum}[\operatorname{SA} 4[a, c, d],\{c, 0,174\}], 601],\{d, 0,174\}], 601]$

The functions TABi [a_] compute $T_{A, B}^{\chi_{i}}(a)$ for $i=1,2,3,4$ :

$\mathrm{TAB} 1\left[\mathrm{a}_{-}\right]:=\operatorname{Mod}[-\mathrm{RA} 1[\mathrm{a}] * 175 * \mathrm{iv}[\mathrm{RB} 1[\mathrm{a}], 61]+\mathrm{a}, 61]$

TAB2 [a_] $:=\operatorname{Mod}[-\operatorname{RA} 2[\mathrm{a}] * 61 * \mathrm{iv}[\mathrm{RB} 2[\mathrm{a}], 1861]+\mathrm{a}, 1861]$

$\mathrm{TAB} 3\left[\mathrm{a}_{-}\right]:=\operatorname{Mod}[-\mathrm{RA} 3[\mathrm{a}] * 175 * \mathrm{iv}[\mathrm{RB} 3[\mathrm{a}], 1861]+\mathrm{a}, 1861]$

TAB4 [a_] $:=\operatorname{Mod}[-\operatorname{RA} 4[\mathrm{a}] * 175 * \mathrm{iv}[\mathrm{RB} 4[\mathrm{a}], 601]+\mathrm{a}, 601]$

The following are needed to compute $E_{\chi_{i}}(r)$ and $F_{\chi_{i}}(r)$ modulo $I_{i}$ :

$\mathrm{A}\left[\mathrm{c}_{-}, \mathrm{d}_{-}, \mathrm{n}_{-}, \mathrm{q}_{-}\right]:=-\mathrm{Floor}[(\mathrm{d}-\mathrm{c} \mathrm{n}) / \mathrm{q}]$

$t\left[c_{-}, d_{-}, n_{-}, q_{-}\right]:=d-c n-q F l o o r[(d-n c) / q]$

$\mathrm{B}\left[\mathrm{c}_{-}, \mathrm{d}_{-}, \mathrm{n}_{-}, \mathrm{q}_{-}\right]:=-\mathrm{F} \operatorname{loor}[(\mathrm{c}-2 \mathrm{nt}[\mathrm{c}, \mathrm{d}, \mathrm{n}, \mathrm{q}]) / \mathrm{q}]$

$e\left[c_{-}, d_{-}, n_{-}, q_{-}\right]:=c-2 n t[c, d, n, q]+q B[c, d, n, q]$

$E\left[c_{-}, d_{-}, n_{-}, q_{-}\right]:=3 c^{\wedge} 2 n+6 d^{\wedge} 2 n-3 e[c, d, n, q] \wedge 2 n$

$-12 A[c, d, n, q] c q+3 c n q-6 d n q+3 e[c, d, n, q] n q$

$+6 A[c, d, n, q] q^{\wedge} 2-6 B[c, d, n, q] q^{\wedge} 2+2 n q^{\wedge} 2$

$+12 B[c, d, n, q] q t[c, d, n, q]-6 n q t[c, d, n, q]$

$-6 \mathrm{n} \mathrm{t}[\mathrm{c}, \mathrm{d}, \mathrm{n}, \mathrm{q}] \sim 2$

$F\left[c_{-}, d_{-}, n_{-}, q_{-}\right]:=-9 c^{\wedge} 2 q+6 d^{\wedge} 2 q-3 e[c, d, n, q] \wedge 2+9 q^{\wedge} 2$

$-6 \mathrm{~d} \mathrm{q}^{\wedge} 2+3 \mathrm{e}[\mathrm{c}, \mathrm{d}, \mathrm{n}, \mathrm{q}] \mathrm{q}^{\wedge} 2+2 \mathrm{q}^{\wedge} 3-18 \mathrm{q}^{\wedge} 2 \mathrm{t}[\mathrm{c}, \mathrm{d}, \mathrm{n}, \mathrm{q}]$

$+18 \mathrm{qt}[\mathrm{c}, \mathrm{d}, \mathrm{n}, \mathrm{q}] \sim 2$

$\operatorname{SF} 1\left[\mathrm{n}_{-}, \mathrm{c}_{-}, \mathrm{d}_{-}\right]:=\operatorname{Mod}\left[\operatorname{ch} 1\left[\mathrm{c}^{\wedge} 2-2 \mathrm{~d}^{\wedge} 2+2 \mathrm{n} \mathrm{c} d\right] * \mathrm{~F}[175, \mathrm{n}, \mathrm{c}, \mathrm{d}], 61\right]$

$\mathrm{SF} 2\left[\mathrm{n}_{-}, \mathrm{c}_{-}, \mathrm{d}_{-}\right]:=\operatorname{Mod}\left[\mathrm{ch} 2\left[\mathrm{c}^{\wedge} 2-2 \mathrm{~d}^{\wedge} 2+2 \mathrm{n} \mathrm{c} \mathrm{d}\right] * \mathrm{~F}[61, \mathrm{n}, \mathrm{c}, \mathrm{d}], 1861\right]$

$\mathrm{SF} 3\left[\mathrm{n}_{-}, \mathrm{c}_{-}, \mathrm{d}_{-}\right]:=\operatorname{Mod}\left[\mathrm{ch} 3\left[\mathrm{c}^{\wedge} 2-2 \mathrm{~d}^{\wedge} 2+2 \mathrm{n} \mathrm{c} d\right] * \mathrm{~F}[175, \mathrm{n}, \mathrm{c}, \mathrm{d}], 1861\right]$

$\mathrm{SF} 4\left[\mathrm{n}_{-}, c_{-}, \mathrm{d}_{-}\right]:=\operatorname{Mod}\left[\operatorname{ch} 4\left[\mathrm{c}^{\wedge} 2-2 \mathrm{~d}^{\wedge} 2+2 \mathrm{n} \mathrm{c} d\right] * \mathrm{~F}[175, \mathrm{n}, \mathrm{c}, \mathrm{d}], 601\right]$

$\operatorname{SE} 1\left[\mathrm{n}_{-}, c_{-}, d_{-}\right]:=\operatorname{Mod}\left[\operatorname{ch} 1\left[c^{\wedge} 2-2 d^{\wedge} 2+2 \mathrm{n} c d\right] * E[175, \mathrm{n}, \mathrm{c}, \mathrm{d}], 61\right]$

$\mathrm{SE} 2\left[\mathrm{n}_{-}, \mathrm{c}_{-}, \mathrm{d}_{-}\right]:=\operatorname{Mod}\left[\mathrm{ch} 2\left[\mathrm{c}^{\wedge} 2-2 \mathrm{~d}^{\wedge} 2+2 \mathrm{n} \mathrm{c} d\right] * \mathrm{E}[61, \mathrm{n}, \mathrm{c}, \mathrm{d}], 1861\right]$

$\operatorname{SE} 3\left[n_{-}, c_{-}, d_{-}\right]:=\operatorname{Mod}\left[\operatorname{ch} 3\left[c^{\wedge} 2-2 d^{\wedge} 2+2 n c^{2} d\right] * E[175, n, c, d], 1861\right]$

$\mathrm{SE} 4\left[\mathrm{n}_{-}, c_{-}, \mathrm{d}_{-}\right]:=\operatorname{Mod}\left[\operatorname{ch} 4\left[\mathrm{c}^{\wedge} 2-2 \mathrm{~d}^{\wedge} 2+2 \mathrm{n} c \mathrm{~d}\right] * \mathrm{E}[175, \mathrm{n}, \mathrm{c}, \mathrm{d}], 601\right]$

The functions REi [a_] and RFi [a_] compute $E_{\chi_{i}}(a)$ and $F_{\chi_{i}}(a)$ modulo $I_{i}$ respectively, where $\mathrm{q}$ is the conductor for the character $\chi_{i}$ for $i=1,2,3,4$ :

$\operatorname{RF} 1\left[\mathrm{a}_{-}\right]:=$

$\operatorname{Mod}[\operatorname{Sum}[\operatorname{Mod}[\operatorname{Sum}[\operatorname{SF} 1[a, c, d],\{c, 0,174\}], 61],\{d, 0,174\}], 61]$ 


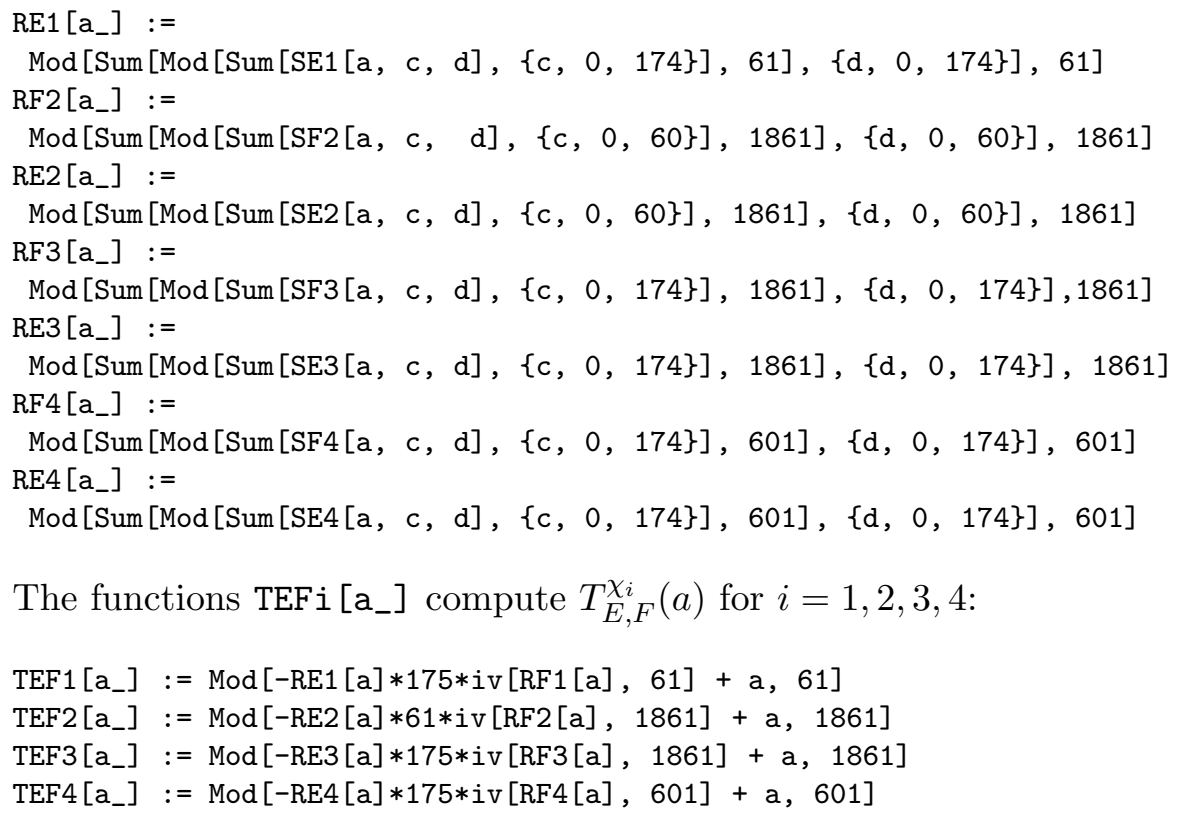

The functions TEFi[a_] compute $T_{E, F}^{\chi_{i}}(a)$ for $i=1,2,3,4$ :

Acknowledgements. The author thanks the referee for helpful suggestions.

This research was supported by Basic Science Research Program through the National Research Foundation of Korea (N.R.F.) funded by the Ministry of Education, Science and Technology 2009-0063182.

\section{References}

[1] A. Biró, Yokoi's conjecture, Acta Arith. 106 (2003), 85-104.

[2] —, Chowla's conjecture, ibid. 107 (2003), 179-194.

[3] D. Byeon and H. Kim, Class number 2 criteria for real quadratic fields of RichaudDegert type, J. Number Theory 62 (1997), 257-272.

[4] -, -, Class number 1 criteria for real quadratic fields of Richaud-Degert type, ibid. 57 (1996), 328-339.

[5] D. Byeon, M. Kim and J. Lee, Mollin's conjecture, Acta Arith. 126 (2007), 99-114.

[6] D. Byeon and J. Lee, Class number 2 problem for certain real quadratic fields of Richaud-Degert type, J. Number Theory 128 (2008), 865-883.

[7] S. Chowla and J. Friedlandler, Class numbers and quadratic residues, Glasgow Math. J. 17 (1976), 47-52.

[8] G. Degert, Über die Bestimmung der Grundeinheit gewisser reell-quadratischer Zahlkörper, Abh. Math. Sem. Univ. Hamburg 22 (1958), 92-97.

[9] G. Janusz, Algebraic Number Fields, Grad. Stud. Math. 7, Amer. Math. Soc., 1996.

[10] R. A. Mollin, Class number one criteria for real quadratic fields. I, Proc. Japan Acad. 63 (1987), 121-125.

[11] -, Quadratics, CRC Press, 1996. 
[12] R. A. Mollin and H. C. Williams, Solution of the class number one problem for real quadratic fields of extended Richaud-Degert type (with one possible exception), in: Number Theory, R. A. Mollin (ed.), de Gruyter, Berlin, 1990, 417-425.

[13] T. Shintani, On evaluation of zeta functions of totally real algebraic number fields at non-positive integers, J. Fac. Sci. Univ. Tokyo 63 (1976), 393-417.

[14] -, On special values of zeta functions of totally real algebraic number fields, in: Proc. Int. Congress Math., Helsinki, 1978, 591-597.

[15] H. Yokoi, Class number one problem for certain kind of real quadratic fields, in: Proc. Int. Conf. (Katata, 1986), Nagoya Univ., Nagoya, 1986, 125-137.

Department of Mathematics

Korea Advanced Institute of Science and Technology

Daejeon, South Korea

E-mail: lee9311@snu.ac.kr

Received on 11.2.2008

and in revised form on 27.4.2009 